\title{
Angular distribution as an effective probe of new physics in semihadronic three-body meson decays
}

\author{
C. S. Kim, ${ }^{1,2, *}$ Seong Chan Park, ${ }^{1, \dagger}$ and Dibyakrupa Sahoo ${ }^{1, *}$ \\ ${ }^{1}$ Department of Physics and IPAP, Yonsei University, Seoul 03722, Korea \\ ${ }^{2}$ Institute of High Energy Physics, Dongshin University, Naju 58245, Korea
}

(Received 20 March 2019; published 9 July 2019)

\begin{abstract}
We analyze, in a fully model-independent manner, the effects of new physics on a few semihadronic three-body meson decays of the type $P_{i} \rightarrow P_{f} f_{1} f_{2}$, where $P_{i}, P_{f}$ are well chosen pseudoscalar mesons and $f_{1,2}$ denote fermions out of which at least one gets detected in experiments. We find that the angular distribution of events of these decays can probe many interesting new physics, such as the nature of the intermediate particle that can cause lepton-flavor violation or presence of heavy sterile neutrino or new intermediate particles or new interactions. We also provide angular asymmetries which can quantify the effects of new physics in these decays. We illustrate the effectiveness of our proposed methodology with a few well chosen decay modes showing how we can probe certain specific new physics possibilities without necessarily worrying about any hadronic uncertainties.
\end{abstract}

DOI: 10.1103/PhysRevD.100.015005

\section{INTRODUCTION}

New physics (NP), or physics beyond the standard model, involves various models that extend the well verified standard model (SM) of particle physics by introducing a number of new particles with novel properties and interactions. Though various aspects of many of these particles and interactions are constrained by existing experimental data, we have yet to detect any definitive signature of new physics in our experiments. Nevertheless, recent experimental studies in $B$ meson decays, such as $B \rightarrow K^{(*)} \ell^{-} \ell^{+}$[1], $B_{s} \rightarrow \phi \ell^{-} \ell^{+}$[2], $B \rightarrow D^{(*)} \ell \nu$ [3], and $B_{c} \rightarrow J / \psi \ell \nu$ [4] (where $\ell$ can be $e, \mu$, or $\tau$ ) have reported anomalous observations raising the expectation of discovery of new physics with more statistical significance. In this context, model-independent studies of such semileptonic three-body meson decay processes become important, as they can identify generic signatures of new physics which can be probed experimentally. In this paper, we have analyzed the effects of new physics, in a modelindependent manner, on the angular distribution of a

\footnotetext{
*cskim@yonsei.ac.kr

Corresponding author. sc.park@yonsei.ac.kr

*Corresponding author. sahoodibya@yonsei.ac.kr
}

Published by the American Physical Society under the terms of the Creative Commons Attribution 4.0 International license. Further distribution of this work must maintain attribution to the author(s) and the published article's title, journal citation, and DOI. Funded by SCOAP. general semihadronic three-body meson decay of the type $P_{i} \rightarrow P_{f} f_{1} f_{2}$, where $P_{i}$ and $P_{f}$ are the initial and final pseudoscalar mesons, respectively, and $f_{1,2}$ denote fermions (which may or may not be leptons but not quarks), out of which at least one gets detected experimentally. The presence of new interactions, or new particles such as fermionic dark matter (DM) particles or heavy sterile neutrinos or long-lived particles (LLP) would leave their signature in the angular distribution, and we show by example how new physics contributions can be quantified from angular asymmetries. Our methodology can be used for detection of new physics in experimental study of various three-body pseudoscalar meson decays at various collider experiments such as LHCb and Belle II.

When the final fermions $f_{1,2}$ are leptons, the decay $P_{i} \rightarrow P_{f} f_{1} f_{2}$ is usually referred to as the semileptonic decay of the meson $P_{i}$. We must emphasize that, in this paper, we consider the possibilities that the fermions $f_{1}$ and $f_{2}$ could be either leptons or nonleptons (which includes exotic fermions such as the ones related to dark matter or long-lived ones), but not quarks. The existing literature is rich in the analysis of semileptonic decays of pseudoscalar mesons for probing the SM and beyond, including important investigations towards estimation of the relevant hadronic form factors. For a review on semileptonic decays of the $B$ meson, we urge the reader to see Ref. [5]. In this paper, we consider not only exotic fermions in the final state but also lepton-flavor violating processes, and we showcase the effectiveness of angular analysis in detecting the effects of new physics. We also show that in certain specific cases one need not worry about hadronic uncertainties. 
The structure of our paper is as follows. In Sec. II, we discuss the most general Lagrangian and amplitude, following Lorentz invariance and including all possible NP contributions to our process under consideration. The relevant details of kinematics is then described in Sec. III. This is followed by a discussion on the angular distribution and the various angular asymmetries in Sec. IV, keeping in mind the usage of the frame-independent Dalitz plot for experimental study. In Sec. VA, we present a classification of all of the decay modes under our consideration. This is followed by a systematic discussion of the effects of new physics on the angular distribution in certain well chosen examples, in Secs. V B and V C, with an emphasis on various aspects that are free from hadronic uncertainties. We also provide an example of equivalent parametrizations of new physics effects. In Sec. VI, we conclude by highlighting the important aspects of our methodology and its possible experimental realization.

\section{MOST GENERAL LAGRANGIAN AND AMPLITUDE}

The process under our consideration is $P_{i} \rightarrow P_{f} f_{1} f_{2}$, where $P_{i, f}$ denote pseudoscalar mesons which can be $B, B_{s}, B_{c}, D, K, \pi$, etc., as appropriate, and $f_{1} f_{2}$ can be $\ell^{-} \ell^{+}, \ell \bar{\ell}^{\prime}, \ell \nu_{\ell}, \ell \nu_{S}, \ell f^{\mathrm{DM}}, \nu_{\ell} \bar{\nu}_{\ell}, \nu_{S} \bar{\nu}_{\ell}, \nu_{\ell} \bar{\nu}_{S}, \nu_{S} \bar{\nu}_{S}$, $f^{\mathrm{DM}} f^{\mathrm{DM}}, f_{1}^{\mathrm{DM}} f_{2}^{\mathrm{DM}}, f_{1}^{\mathrm{LLP}} f_{2}^{\mathrm{LLP}}$ (with $\ell, \ell^{\prime}=e, \mu, \tau$ denoting leptons, $\nu_{S}$ being sterile neutrino, $f_{1,2}^{\mathrm{DM}}$ as fermionic dark matter, and $f_{1,2}^{\mathrm{LLP}}$ as long-lived fermions). It is clear that our analysis must be an all encompassing formulation by which we can analyze not only processes such as $B \rightarrow D \ell \nu$ that are readily favored in the SM but also SM allowed rare decays such as $B \rightarrow K \ell^{-} \ell^{+}$and $B \rightarrow D \ell^{-} \ell^{+}$as well as the SM forbidden lepton-flavor violating processes such as $B \rightarrow P \ell^{ \pm} \ell^{\prime \mp}$, where $P=\pi, K, D, \ell \neq \ell^{\prime}$, and $\ell, \ell^{\prime}=e, \mu, \tau$. Therefore, our analysis has to be fully model independent and general in nature. If we consider some specific NP model, leptoquark models as an example, then we can allow interaction vertices where quarks transform to leptons, and vice versa. In such a case, the fundamental description of the underlying process would include quark and lepton fields together in every individual current. However, by using Fierz transformations, we can rewrite the product of these currents as a linear combination of products of currents involving purely lepton fields and purely quark fields. Finally, the quark-level description would give rise to effective hadronic currents with appropriate form factors which are estimated in many different ways, such as by using the heavy quark effective theory [6], the lattice QCD [7], QCD light-cone sum rule [8], or the covariant confined quark model [9], etc. Since we are analyzing a diverse set of meson decays in a unified formalism, we shall refrain from delving deeply into the details of the form factors involved in any specific decay mode. Considering the process $P_{i} \rightarrow P_{f} f_{1} f_{2}$ and applying the Fierz transformations where necessary, we can write down the most general form of the effective Lagrangian as follows,

$$
\begin{aligned}
\mathcal{L}_{\text {eff }}= & J_{S}\left(\bar{f}_{1} f_{2}\right)+J_{P}\left(\bar{f}_{1} \gamma^{5} f_{2}\right)+\left(J_{V}\right)_{\alpha}\left(\bar{f}_{1} \gamma^{\alpha} f_{2}\right) \\
& +\left(J_{A}\right)_{\alpha}\left(\bar{f}_{1} \gamma^{\alpha} \gamma^{5} f_{2}\right)+\left(J_{T_{1}}\right)_{\alpha \beta}\left(\bar{f}_{1} \sigma^{\alpha \beta} f_{2}\right) \\
& +\left(J_{T_{2}}\right)_{\alpha \beta}\left(\bar{f}_{1} \sigma^{\alpha \beta} \gamma^{5} f_{2}\right)+\text { H.c. },
\end{aligned}
$$

where $J_{S}, J_{P},\left(J_{V}\right)_{\alpha},\left(J_{A}\right)_{\alpha},\left(J_{T_{1}}\right)_{\alpha \beta},\left(J_{T_{2}}\right)_{\alpha \beta}$ are the different hadronic currents which effectively describe the quark-level transitions from $P_{i}$ to $P_{f}$ meson. ${ }^{1}$ It should be noted that we have kept both the $\sigma^{\alpha \beta}$ and $\sigma^{\alpha \beta} \gamma^{5}$ terms. This is because of the fact that the currents $\bar{f}_{1} \sigma^{\alpha \beta} f_{2}$ and $\bar{f}_{1} \sigma^{\alpha \beta} \gamma^{5} f_{2}$ describe two different physics aspects-namely, the magnetic dipole and electric dipole contributions, respectively. In the SM, vector and axial-vector currents (mediated by photon, $W^{ \pm}$, and $Z^{0}$ bosons) and the scalar current (mediated by Higgs boson) contribute. So every other term in Eq. (1) except the ones with $J_{S},\left(J_{V}\right)_{\alpha}$, and $\left(J_{A}\right)_{\alpha}$ can appear in some specific NP model. Since, in this paper, we want to concentrate on a fully model-independent analysis to get generic signatures of new physics, we shall refrain from venturing into details of any specific NP model, which nevertheless are also useful. It is important to note that $J_{S},\left(J_{V}\right)_{\alpha}$, and $\left(J_{A}\right)_{\alpha}$ can also get modified due to NP contributions.

In order to get the most general amplitude for our process under consideration, we need to go from the effective quark-level description of Eq. (1) to the mesonlevel description by defining appropriate form factors. It is easy to write down the most general form of the amplitude for the process $P_{i} \rightarrow P_{f} f_{1} f_{2}$ depicted in Fig. 1 as follows,

$$
\begin{aligned}
\mathcal{M}\left(P_{i} \rightarrow P_{f} f_{1} f_{2}\right)= & F_{S}\left(\bar{f}_{1} f_{2}\right)+F_{P}\left(\bar{f}_{1} \gamma^{5} f_{2}\right) \\
& +\left(F_{V}^{+} p_{\alpha}+F_{V}^{-} q_{\alpha}\right)\left(\bar{f}_{1} \gamma^{\alpha} f_{2}\right) \\
& +\left(F_{A}^{+} p_{\alpha}+F_{A}^{-} q_{\alpha}\right)\left(\bar{f}_{1} \gamma^{\alpha} \gamma^{5} f_{2}\right) \\
& +F_{T_{1}} p_{\alpha} q_{\beta}\left(\bar{f}_{1} \sigma^{\alpha \beta} f_{2}\right) \\
& +F_{T_{2}} p_{\alpha} q_{\beta}\left(\bar{f}_{1} \sigma^{\alpha \beta} \gamma^{5} f_{2}\right),
\end{aligned}
$$

\footnotetext{
${ }^{1}$ The subscripts $S, P, V, A, T$ in the hadronic currents denote the fact that the associated external fermionic currents involving the $f_{1}, f_{2}$ fields are of scalar, pseudoscalar, vector, axial vector, and tensor type, respectively. For the hadronic currents and subsequently for the effective form factors, we have followed the same notation as in Ref. [10]. Since Eq. (1) involves all of the Dirac bilinears, it does describe effectively all possible interactions that preserve Lorentz invariance.
} 


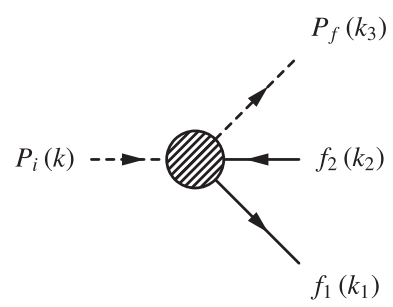

FIG. 1. Feynman diagram for $P_{i} \rightarrow P_{f} f_{1} f_{2}$ considering $f_{1}$ as a particle and $f_{2}$ as an antiparticle. Here the blob denotes the effective vertex and includes contributions from all of the form factors defined in Eq. (3).

where $F_{S}, F_{P}, F_{V}^{ \pm}, F_{A}^{ \pm}, F_{T_{1}}$, and $F_{T_{2}}$ are the relevant form factors $^{2}$ and are defined as follows,

$$
\begin{aligned}
\left\langle P_{f}\left|J_{S}\right| P_{i}\right\rangle & =F_{S}, \\
\left\langle P_{f}\left|J_{P}\right| P_{i}\right\rangle & =F_{P}, \\
\left\langle P_{f}\left|\left(J_{V}\right)_{\alpha}\right| P_{i}\right\rangle & =F_{V}^{+} p_{\alpha}+F_{V}^{-} q_{\alpha}, \\
\left\langle P_{f}\left|\left(J_{A}\right)_{\alpha}\right| P_{i}\right\rangle & =F_{A}^{+} p_{\alpha}+F_{A}^{-} q_{\alpha}, \\
\left\langle P_{f}\left|\left(J_{T_{1}}\right)_{\alpha \beta}\right| P_{i}\right\rangle & =F_{T_{1}} p_{\alpha} q_{\beta}, \\
\left\langle P_{f}\left|\left(J_{T_{2}}\right)_{\alpha \beta}\right| P_{i}\right\rangle & =F_{T_{2}} p_{\alpha} q_{\beta},
\end{aligned}
$$

with $p \equiv k+k_{3}$ and $q \equiv k-k_{3}=k_{1}+k_{2}$, in which $k, k_{1}, k_{2}, k_{3}$ are the 4-momenta of $P_{i}, f_{1}, f_{2}$, and $P_{f}$, respectively (see Fig. 1). All of the form factors appearing in the amplitude in Eq. (2) and as defined in Eq. (3) are, in general, complex and contain all NP information. It should be noted that for brevity of expression to follow, we have implicitly put all of the relevant Cabibbo-KobayashiMaskawa matrix elements as well as coupling constants and propagators inside the definitions of these form factors. Moreover, these form factors also implicitly include contributions from both the short-distance $(\mathrm{QCD}$, weak interaction, and NP) effects and long-distance QCD effects. Detailed study of how NP affects the form factors is very

\footnotetext{
${ }^{2}$ These eight form factors form a complete set of "effective" form factors. As an example, let us consider the decay $B \rightarrow K \mu^{-} \mu^{+}$, which is very well studied in the literature. The underlying quark-level transition is $b \rightarrow s \mu^{-} \mu^{+}$, which involves flavor-changing neutral current and is, therefore, not allowed at tree level in the SM. However, the decay does happen at loop level via the famous penguin diagrams. For a detailed study of the underlying physics in this decay, see Ref. [11]. From the references in Ref. [12], we can see that, at the meson level, the hadronic current (equivalently, hadronic matrix element) in the SM can be decomposed into two components, one involving $p_{\alpha}$ and another involving $q_{\alpha}$, which can be compared with what we have in Eq. (3) for the vector and axial-vector cases.
}

helpful while considering specific decay modes and specific NP models. It must also be noted that all of the form factors have an implicit dependence on $q^{2}$ which is the invariant mass of the $f_{1} f_{2}$ system. Since in our analysis we are not going to consider the explicit variation of our observables with respect to $q^{2}$, we shall refrain from discussing any details of the form factors. This helps us to keep our analysis fully model independent. In the SM and for our decays under consideration, only $F_{S}, F_{V}^{ \pm}$, and $F_{A}^{ \pm}$are present. The presence of NP can modify these as well as introduce other form factors. These various NP contributions would leave behind their signatures in the angular distribution, for which we need to specify the kinematics in a chosen frame of reference.

\section{DECAY KINEMATICS}

We shall consider the decay $P_{i} \rightarrow P_{f} f_{1} f_{2}$ in the Gottfried-Jackson frame, especially the center-of-momentum frame of the $f_{1}, f_{2}$ system, which is shown in Fig. 2. In this frame, the parent meson $P_{i}$ flies along the positive $z$ direction with 4-momentum $k=(E, \mathbf{k})=(E, 0,0,|\mathbf{k}|)$ and decays to the daughter meson $P_{f}$, which also flies along the positive $z$ direction with 4-momentum $k_{3}=\left(E_{3}, \mathbf{k}_{3}\right)=\left(E_{3}, 0,0,\left|\mathbf{k}_{3}\right|\right)$, and to $f_{1}, f_{2}$, which fly away back to back with 4-momenta $k_{1}=\left(E_{1}, \mathbf{k}_{1}\right)$ and $k_{2}=\left(E_{2}, \mathbf{k}_{2}\right)$, respectively, such that by conservation of 4-momentum we get $\mathbf{k}_{1}+\mathbf{k}_{2}=\mathbf{0}, \mathbf{k}=\mathbf{k}_{3}$, and $E=E_{1}+E_{2}+E_{3}$. The fermion $f_{1}$ (which we assume can be observed experimentally) flies out subtending an angle $\theta$ with respect to the direction of flight of the $P_{i}$ meson in this Gottfried-Jackson frame. The three invariant mass squares involved in the decay under consideration are defined as follows,

$$
\begin{aligned}
& s=\left(k_{1}+k_{2}\right)^{2}=\left(k-k_{3}\right)^{2}, \\
& t=\left(k_{1}+k_{3}\right)^{2}=\left(k-k_{2}\right)^{2}, \\
& u=\left(k_{2}+k_{3}\right)^{2}=\left(k-k_{1}\right)^{2} .
\end{aligned}
$$

It is easy to show that $s+t+u=m_{i}^{2}+m_{f}^{2}+m_{1}^{2}+m_{2}^{2}$, where $m_{i}, m_{f}, m_{1}$, and $m_{2}$ denote the masses of particles

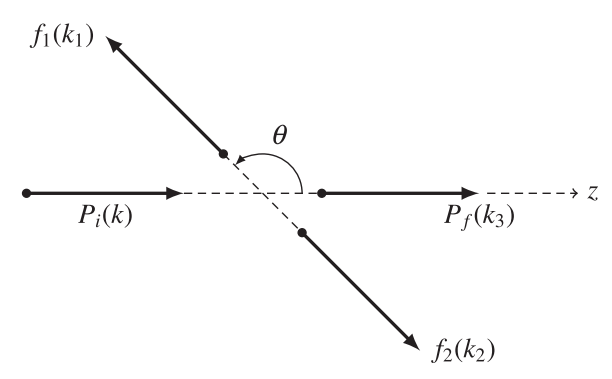

FIG. 2. Decay of $P_{i} \rightarrow P_{f} f_{1} f_{2}$ in the Gottfried-Jackson frame. 
$P_{i}, P_{f}, f_{1}$, and $f_{2}$, respectively. In the Gottfried-Jackson frame, the expressions for $t$ and $u$ are given by

$$
\begin{gathered}
t=a_{t}-b \cos \theta, \\
u=a_{u}+b \cos \theta,
\end{gathered}
$$

where

$$
\begin{aligned}
a_{t} & =m_{1}^{2}+m_{f}^{2}+\frac{1}{2 s}\left(s+m_{1}^{2}-m_{2}^{2}\right)\left(m_{i}^{2}-m_{f}^{2}-s\right), \\
a_{u} & =m_{2}^{2}+m_{f}^{2}+\frac{1}{2 s}\left(s-m_{1}^{2}+m_{2}^{2}\right)\left(m_{i}^{2}-m_{f}^{2}-s\right), \\
b & =\frac{1}{2 s} \sqrt{\lambda\left(s, m_{1}^{2}, m_{2}^{2}\right) \lambda\left(s, m_{i}^{2}, m_{f}^{2}\right)},
\end{aligned}
$$

with the Källén function $\lambda(x, y, z)$ defined as

$$
\lambda(x, y, z)=x^{2}+y^{2}+z^{2}-2(x y+y z+z x) .
$$

It is clear that $a_{t}, a_{u}$, and $b$ are functions of $s$ only. For the special case of $m_{1}=m_{2}=m$ (say), we have $a_{t}=a_{u}=$ $\frac{1}{2}\left(m_{i}^{2}+m_{f}^{2}+2 m^{2}-s\right)$ and $b=\frac{1}{2} \sqrt{\left(1-4 m^{2} / s\right) \lambda\left(s, m_{i}^{2}, m_{f}^{2}\right)}$. It is important to note that we shall use the angle $\theta$ in our angular distribution.

\section{MOST GENERAL ANGULAR DISTRIBUTION AND ANGULAR ASYMMETRIES}

Considering the amplitude given in Eq. (2), the most general angular distribution in the Gottfried-Jackson frame is given by

$$
\frac{d^{2} \Gamma}{d s d \cos \theta}=\frac{b \sqrt{s}\left(C_{0}+C_{1} \cos \theta+C_{2} \cos ^{2} \theta\right)}{128 \pi^{3} m_{i}^{2}\left(m_{i}^{2}-m_{f}^{2}+s\right)},
$$

where $C_{0}, C_{1}$, and $C_{2}$ are functions of $s$ and are given by

$$
\begin{aligned}
& C_{0}=2\left(-\left|F_{T_{1}}\right|^{2}\left(-\Sigma m_{12}^{2} s^{2}+2 \Sigma m_{12}^{2}\left(\Sigma m^{2}\right)_{i f} s+\left(\Delta m^{2}\right)_{12}^{2} s-\Delta a_{t u}^{2} s\right.\right. \\
& \left.-2\left(\Delta m^{2}\right)_{12}^{2}\left(\Sigma m^{2}\right)_{i f}-\left(\Delta m^{2}\right)_{i f}^{2} \Sigma m_{12}^{2}+2 \Delta a_{t u}\left(\Delta m^{2}\right)_{12}\left(\Delta m^{2}\right)_{i f}\right) \\
& -2 \operatorname{Im}\left(F_{V}^{+} F_{T_{1}}^{*}\right)\left(-\Sigma m_{12} s^{2}+2 \Sigma m_{12}\left(\Sigma m^{2}\right)_{i f} s+\Delta m_{12}\left(\Delta m^{2}\right)_{12} s\right. \\
& \left.-2 \Delta m_{12}\left(\Delta m^{2}\right)_{12}\left(\Sigma m^{2}\right)_{i f}-\left(\Delta m^{2}\right)_{i f}^{2} \Sigma m_{12}+\Delta a_{t u} \Delta m_{12}\left(\Delta m^{2}\right)_{i f}\right) \\
& +\left|F_{T_{2}}\right|^{2}\left(\Delta m_{12}^{2} s^{2}-2 \Delta m_{12}^{2}\left(\Sigma m^{2}\right)_{i f} s-\left(\Delta m^{2}\right)_{12}^{2} s+\Delta a_{t u}^{2} s+2\left(\Delta m^{2}\right)_{12}^{2}\left(\Sigma m^{2}\right)_{i f}\right. \\
& \left.+\Delta m_{12}^{2}\left(\Delta m^{2}\right)_{i f}^{2}-2 \Delta a_{t u}\left(\Delta m^{2}\right)_{12}\left(\Delta m^{2}\right)_{i f}\right) \\
& -2 \operatorname{Im}\left(F_{A}^{+} F_{T_{2}}^{*}\right)\left(\Delta m_{12} s^{2}-2 \Delta m_{12}\left(\Sigma m^{2}\right)_{i f} s-\left(\Delta m^{2}\right)_{12} \Sigma m_{12} s\right. \\
& \left.+2\left(\Delta m^{2}\right)_{12} \Sigma m_{12}\left(\Sigma m^{2}\right)_{i f}-\Delta a_{t u}\left(\Delta m^{2}\right)_{i f} \Sigma m_{12}+\Delta m_{12}\left(\Delta m^{2}\right)_{i f}^{2}\right) \\
& +\left|F_{A}^{+}\right|^{2}\left(s^{2}-2\left(\Sigma m^{2}\right)_{i f} s-\Sigma m_{12}^{2} s+2 \Sigma m_{12}^{2}\left(\Sigma m^{2}\right)_{i f}+\left(\Delta m^{2}\right)_{i f}^{2}-\Delta a_{t u}^{2}\right) \\
& +\left|F_{V}^{+}\right|^{2}\left(s^{2}-2\left(\Sigma m^{2}\right)_{i f} s-\Delta m_{12}^{2} s+2 \Delta m_{12}^{2}\left(\Sigma m^{2}\right)_{i f}+\left(\Delta m^{2}\right)_{i f}^{2}-\Delta a_{t u}^{2}\right) \\
& +\left|F_{A}^{-}\right|^{2}\left(\Sigma m_{12}^{2} s-\left(\Delta m^{2}\right)_{12}^{2}\right)-2 \operatorname{Re}\left(F_{P} F_{A}^{-*}\right)\left(\Sigma m_{12} s-\Delta m_{12}\left(\Delta m^{2}\right)_{12}\right) \\
& -\left|F_{V}^{-}\right|^{2}\left(\left(\Delta m^{2}\right)_{12}^{2}-\Delta m_{12}^{2} s\right)-2 \operatorname{Re}\left(F_{S} F_{V}^{-*}\right)\left(\left(\Delta m^{2}\right)_{12} \Sigma m_{12}-\Delta m_{12} s\right) \\
& -\left|F_{S}\right|^{2}\left(\Sigma m_{12}^{2}-s\right)-\left|F_{P}\right|^{2}\left(\Delta m_{12}^{2}-s\right)+2 \operatorname{Re}\left(F_{A}^{+} F_{A}^{-*}\right)\left(\left(\Delta m^{2}\right)_{i f} \Sigma m_{12}^{2}-\Delta a_{t u}\left(\Delta m^{2}\right)_{12}\right) \\
& -2 \operatorname{Re}\left(F_{P} F_{A}^{+*}\right)\left(\left(\Delta m^{2}\right)_{i f} \Sigma m_{12}-\Delta a_{t u} \Delta m_{12}\right)-2 \operatorname{Re}\left(F_{S} F_{V}^{+*}\right)\left(\Delta a_{t u} \Sigma m_{12}-\Delta m_{12}\left(\Delta m^{2}\right)_{i f}\right) \\
& \left.+2 \operatorname{Re}\left(F_{V}^{+} F_{V}^{-*}\right)\left(\Delta m_{12}^{2}\left(\Delta m^{2}\right)_{i f}-\Delta a_{t u}\left(\Delta m^{2}\right)_{12}\right)\right) \text {, } \\
& C_{1}=8 b\left(\Delta m_{12}\left(\operatorname{Im}\left(F_{V}^{-} F_{T_{1}}^{*}\right) s-\operatorname{Re}\left(F_{P} F_{A}^{+*}\right)\right)+\Sigma m_{12}\left(-\operatorname{Im}\left(F_{A}^{-} F_{T_{2}}^{*}\right) s+\operatorname{Re}\left(F_{S} F_{V}^{+*}\right)\right.\right. \\
& \left.-\left(\Delta m^{2}\right)_{i f} \operatorname{Im}\left(F_{A}^{+} F_{T_{2}}^{*}\right)\right)+\Delta a_{t u}\left(\left|F_{V}^{+}\right|^{2}+\left|F_{A}^{+}\right|^{2}\right)+\left(\operatorname{Im}\left(F_{S} F_{T_{1}}^{*}\right)+\operatorname{Im}\left(F_{P} F_{T_{2}}^{*}\right)\right) s \\
& \left.+\left(\Delta m^{2}\right)_{12}\left(\operatorname{Re}\left(F_{V}^{+} F_{V}^{-*}\right)+\operatorname{Re}\left(F_{A}^{+} F_{A}^{-*}\right)\right)+\left(\Delta m^{2}\right)_{i f} \Delta m_{12} \operatorname{Im}\left(F_{V}^{+} F_{T_{1}}^{*}\right)\right), \\
& C_{2}=8 b^{2}\left(\left(\left|F_{T_{2}}\right|^{2}+\left|F_{T_{1}}\right|^{2}\right) s-\left|F_{V}^{+}\right|^{2}-\left|F_{A}^{+}\right|^{2}\right),
\end{aligned}
$$


with $\Delta a_{t u}=a_{t}-a_{u}, \Delta m_{12}=m_{1}-m_{2}, \Delta m_{i f}=m_{i}-m_{f}$, $\Sigma m_{12}=m_{1}+m_{2}, \Sigma m_{i f}=m_{i}+m_{f},\left(\Delta m^{2}\right)_{12}=\Delta m_{12} \Sigma m_{12}=$ $m_{1}^{2}-m_{2}^{2}, \quad\left(\Delta m^{2}\right)_{i f}=\Delta m_{i f} \Sigma m_{i f}=m_{i}^{2}-m_{f}^{2}, \quad\left(\Sigma m^{2}\right)_{i f}=$ $m_{i}^{2}+m_{f}^{2}$. In the limit $m_{1}=m_{2}$, which happens when $f_{1} f_{2}=\ell^{-} \ell^{+}, \nu \bar{\nu}, f^{\mathrm{DM}} \bar{f}^{\mathrm{DM}}$, etc., our expressions for the angular distribution matches with the corresponding expression in Ref. [10]. It is important to remember that in the SM we come across scalar, vector, and axial vector currents only. Therefore, in the $\mathrm{SM}, F_{P}^{\mathrm{SM}}=F_{T_{1}}^{\mathrm{SM}}=$ $F_{T_{2}}^{\mathrm{SM}}=0$, which implies that,

$$
\begin{aligned}
C_{0}^{\mathrm{SM}}= & 2\left(\left|\left(F_{A}^{+}\right)_{\mathrm{SM}}\right|^{2}\left(s^{2}-2\left(\Sigma m^{2}\right)_{i f} s-\Sigma m_{12}^{2} s+2 \Sigma m_{12}^{2}\left(\Sigma m^{2}\right)_{i f}+\left(\Delta m^{2}\right)_{i f}^{2}-\Delta a_{t u}^{2}\right)\right. \\
& +\left|\left(F_{V}^{+}\right)_{\mathrm{SM}}\right|^{2}\left(s^{2}-2\left(\Sigma m^{2}\right)_{i f} s-\Delta m_{12}^{2} s+2 \Delta m_{12}^{2}\left(\Sigma m^{2}\right)_{i f}+\left(\Delta m^{2}\right)_{i f}^{2}-\Delta a_{t u}^{2}\right) \\
& +\left|\left(F_{A}^{-}\right)_{\mathrm{SM}}\right|^{2}\left(\Sigma m_{12}^{2} s-\left(\Delta m^{2}\right)_{12}^{2}\right)-\left|\left(F_{V}^{-}\right)_{\mathrm{SM}}\right|^{2}\left(\left(\Delta m^{2}\right)_{12}^{2}-\Delta m_{12}^{2} s\right)-\left|\left(F_{S}\right)_{\mathrm{SM}}\right|^{2}\left(\Sigma m_{12}^{2}-s\right) \\
& +2 \operatorname{Re}\left(\left(F_{A}^{+}\right)_{\mathrm{SM}}\left(F_{A}^{-}\right)_{\mathrm{SM}}^{*}\right)\left(\left(\Delta m^{2}\right)_{i f} \Sigma m_{12}^{2}-\Delta a_{t u}\left(\Delta m^{2}\right)_{12}\right) \\
& \left.+2 \operatorname{Re}\left(\left(F_{V}^{+}\right)_{\mathrm{SM}}\left(F_{V}^{-}\right)_{\mathrm{SM}}^{*}\right)\left(\left(\Delta m^{2}\right)_{i f} \Delta m_{12}^{2}-\Delta a_{t u}\left(\Delta m^{2}\right)_{12}\right)\right), \\
C_{1}^{\mathrm{SM}}= & 8 b\left(\Delta a_{t u}\left(\left|\left(F_{V}^{+}\right)_{\mathrm{SM}}\right|^{2}+\left|\left(F_{A}^{+}\right)_{\mathrm{SM}}\right|^{2}\right)+\left(\Delta m^{2}\right)_{12}\left(\operatorname{Re}\left(\left(F_{V}^{+}\right)_{\mathrm{SM}}\left(F_{V}^{-}\right)_{\mathrm{SM}}^{*}\right)+\operatorname{Re}\left(\left(F_{A}^{+}\right)_{\mathrm{SM}}\left(F_{A}^{-}\right)_{\mathrm{SM}}^{*}\right)\right)\right), \\
C_{2}^{\mathrm{SM}}= & -8 b^{2}\left(\left|\left(F_{V}^{+}\right)_{\mathrm{SM}}\right|^{2}+\left|\left(F_{A}^{+}\right)_{\mathrm{SM}}\right|^{2}\right) .
\end{aligned}
$$

It is interesting to note that in the special case of $m_{1}=m_{2}$, such as in $P_{i} \rightarrow P_{f} \ell^{-} \ell^{+}$, we always have $C_{1}^{\mathrm{SM}}=0$. For specific meson decays of the form $P_{i} \rightarrow$ $P_{f} f_{1} f_{2}$ allowed in the SM, one can write down $\left(F_{S}\right)_{\mathrm{SM}}$, $\left(F_{V}^{ \pm}\right)_{\mathrm{SM}}$, and $\left(F_{A}^{ \pm}\right)_{\mathrm{SM}}$, at least in principle. ${ }^{3}$ The SM prediction for the angular distribution can thus be compared with the corresponding experimental measurement. In order to quantitatively compare the theoretical prediction with the experimental measurement, we define the following three angular asymmetries, which can precisely probe $C_{0}, C_{1}$, and $C_{2}$ individually,

$$
\begin{aligned}
A_{0} \equiv A_{0}(s) & =\frac{-\frac{1}{6}\left(\int_{-1}^{-1 / 2}-7 \int_{-1 / 2}^{+1 / 2}+\int_{+1 / 2}^{+1}\right) \frac{d^{2} \Gamma}{d s d \cos \theta} d \cos \theta}{d \Gamma / d s} \\
& =\frac{3 C_{0}}{\left(6 C_{0}+2 C_{2}\right)}, \\
A_{1} \equiv A_{1}(s) & =\frac{-\left(\int_{-1}^{0}-\int_{0}^{+1}\right) \frac{d^{2} \Gamma}{d s d \cos \theta} d \cos \theta}{d \Gamma / d s} \\
& =\frac{3 C_{1}}{\left(6 C_{0}+2 C_{2}\right)}, \\
A_{2} \equiv A_{2}(s) & =\frac{2\left(\int_{-1}^{-1 / 2}-\int_{-1 / 2}^{+1 / 2}+\int_{+1 / 2}^{+1}\right) \frac{d^{2} \Gamma}{d s d \cos \theta} d \cos \theta}{d \Gamma / d s} \\
& =\frac{3 C_{2}}{\left(6 C_{0}+2 C_{2}\right)} .
\end{aligned}
$$

\footnotetext{
${ }^{3}$ In the case of decays mediated by $W^{ \pm}$, the form factors $\left(F_{V}^{ \pm}\right)_{\mathrm{SM}}$ and $\left(F_{A}^{ \pm}\right)_{\mathrm{SM}}$ are related by $\left(F_{V}^{ \pm}\right)_{\mathrm{SM}}=-\left(F_{A}^{ \pm}\right)_{\mathrm{SM}}$ and $\left(F_{V}^{+} / F_{A}^{+}\right)_{\mathrm{SM}}=\left(F_{V}^{-} / F_{A}^{-}\right)_{\mathrm{SM}}$. However, for neutral current mediated decays, $\left(F_{V}^{ \pm}\right)_{\mathrm{SM}} \neq-\left(F_{A}^{ \pm}\right)_{\mathrm{SM}}($ in general $)$, but $\left(F_{V}^{+} / F_{A}^{+}\right)_{\mathrm{SM}}=$ $\left(F_{V}^{-} / F_{A}^{-}\right)_{\mathrm{SM}}$ still remains valid.
}

The angular asymmetries of Eq. (10) are functions of $s$, and it is easy to show that $A_{2}=3\left(1 / 2-A_{0}\right)$. We can do the integration over $s$ in Eq. (7) and define the following normalized angular distribution,

$$
\frac{1}{\Gamma} \frac{d \Gamma}{d \cos \theta}=T_{0}+T_{1} \cos \theta+T_{2} \cos ^{2} \theta
$$

where

$$
T_{j}=3 c_{j} /\left(6 c_{0}+2 c_{2}\right)
$$

for $j=0,1,2$, and with

$$
c_{j}=\int_{\left(m_{1}+m_{2}\right)^{2}}^{\left(m_{i}-m_{f}\right)^{2}} \frac{b \sqrt{s} C_{j}}{128 \pi^{3} m_{i}^{2}\left(m_{i}^{2}-m_{f}^{2}+s\right)} d s
$$

From Eq. (12), it is easy to show that $T_{2}=3\left(1 / 2-T_{0}\right)$, which also ensures that integration over $\cos \theta$ on Eq. (11) is equal to 1 . It is interesting to note that the angular distribution of Eq. (11) can be written in terms of the orthogonal Legendre polynomials of $\cos \theta$ as well,

$$
\frac{1}{\Gamma} \frac{d \Gamma}{d \cos \theta}=\sum_{i=0}^{2}\left\langle G^{(i)}\right\rangle P_{i}(\cos \theta) .
$$

Here we have followed the notation of Ref. [13], which also analyzes decays of the type $P_{i} \rightarrow P_{f} f_{1} f_{2}$, with only leptons for $f_{1,2}$, in a model-independent manner but using a generalized helicity amplitude method. The observables $\left\langle G^{(i)}\right\rangle$ of Eq. (14) are related to $T_{0}, T_{1}$, and $T_{2}$ of Eq. (11) as follows, 


$$
\begin{aligned}
& \left\langle G^{(0)}\right\rangle=T_{0}+T_{2} / 3=1 / 2, \\
& \left\langle G^{(1)}\right\rangle=T_{1}, \\
& \left\langle G^{(2)}\right\rangle=2 T_{2} / 3 .
\end{aligned}
$$

These angular observables $\left\langle G^{(i)}\right\rangle$ can be obtained by using the method of moments $[13,14]$. Another important way to describe the normalized angular distribution is by using a flat term $F_{H} / 2$ and the forward-backward asymmetry $A_{F B}$ [12] as follows,

$\frac{1}{\Gamma} \frac{d \Gamma}{d \cos \theta}=\frac{1}{2} F_{H}+A_{F B} \cos \theta+\frac{3}{4}\left(1-F_{H}\right)\left(1-\cos ^{2} \theta\right)$.

This form of angular distribution has also been used in the experimental community [15] in the study of $B \rightarrow K \ell^{-} \ell^{+}$. The parameters $F_{H}$ and $A_{F B}$ are related to $T_{0}, T_{1}$, and $T_{2}$ as follows,

$$
\begin{aligned}
F_{H} & =2\left(T_{0}+T_{2}\right)=3-4 T_{0}, \\
A_{F B} & =T_{1} .
\end{aligned}
$$

Thus we have shown that Eqs. (11), (14), and (16) are equivalent to one another. In this paper, we choose to work using the normalized angular distribution in terms of $T_{0}$, $T_{1}$, and $T_{2}$, as shown in Eq. (11). This is because the terms $T_{0}, T_{1}$, and $T_{2}$ can be easily determined experimentally by using the $t$-vs- $u$ Dalitz plot, which does not depend on any specific frame of reference. This Dalitz plot can be easily divided into four segments, I, II, III, and IV, as shown in Fig. 3. The segments are decided as follows,

Segment I: $-1 \leq \cos \theta \leq-0.5$,

Segment II: $-0.5<\cos \theta \leq 0$,

Segment II : $0<\cos \theta \leq 0.5$,

Segment IV : $0.5<\cos \theta \leq 1$.

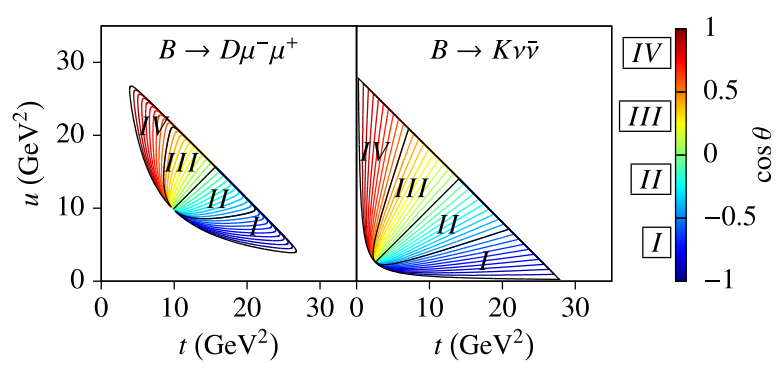

FIG. 3. Two examples depicting the variation of $\cos \theta$ in the interior region of the $t$-vs- $u$ Dalitz plot. The interior of the Dalitz plot can be divided into four segments, I, II, III, and IV, as shown here.
The terms $T_{0}, T_{1}$, and $T_{2}$ can thus be expressed in terms of the following asymmetries,

$$
\begin{aligned}
& T_{0}=-\frac{1}{6}\left(\frac{N_{I}-7\left(N_{I I}+N_{I I I}\right)+N_{I V}}{N_{I}+N_{I I}+N_{I I I}+N_{I V}}\right), \\
& T_{1}=\frac{\left(N_{\mathrm{I}}+N_{\mathrm{II}}\right)-\left(N_{\mathrm{III}}+N_{\mathrm{IV}}\right)}{N_{\mathrm{I}}+N_{\mathrm{II}}+N_{\mathrm{III}}+N_{\mathrm{IV}}} \\
& T_{2}=2\left(\frac{N_{\mathrm{I}}-\left(N_{\mathrm{II}}+N_{\mathrm{III}}\right)+N_{\mathrm{IV}}}{N_{\mathrm{I}}+N_{\mathrm{II}}+N_{\mathrm{III}}+N_{\mathrm{IV}}}\right)
\end{aligned}
$$

where $N_{j}$ denotes the number of events contained in the segment $j$. Since the $t$-vs- $u$ Dalitz plot does not depend on the frame of reference, we need not constraint ourselves to the Gottfried-Jackson frame of Fig. 2, and we can work in the laboratory frame as well. Furthermore, we can use the expressions in Eq. (18) to search for NP.

\section{ILLUSTRATING THE EFFECTS OF NEW PHYSICS ON THE ANGULAR DISTRIBUTION}

\section{A. Classification of the $\boldsymbol{P}_{\boldsymbol{i}} \rightarrow \boldsymbol{P}_{f} f_{1} \boldsymbol{f}_{\mathbf{2}}$ decays}

It should be emphasized that, for our methodology to work, we need to know the angle $\theta$ in the Gottfried-Jackson frame, or equivalently the $t$-vs- $u$ Dalitz plot, which demand that the 4-momenta of the final particles be fully known. Usually, the 4-momenta of the initial and final pseudoscalar mesons are directly measured experimentally. However, depending on the detection possibilities of $f_{1}$ and $f_{2}$, we can identify three distinct scenarios for our process $P_{i} \rightarrow P_{f} f_{1} f_{2}$. We introduce the notations $f_{i}^{J}$ and $f_{i}^{x}$ to denote whether the fermion $f_{i}$ gets detected $(\boldsymbol{})$ or not $(\boldsymbol{X})$ by the detector. Using this notation, the three scenarios are described as follows.

(S1) $P_{i} \rightarrow P_{f}+f_{1}^{\mathcal{V}}+f_{2}^{\mathcal{J}} \equiv P_{f}+$ "visible". Here both $f_{1}$ and $f_{2}$ are detected, e.g., when $f_{1} f_{2}=\ell^{-} \ell^{+}$ or $\ell \bar{\ell}^{\prime}$.

(S2) $P_{i} \rightarrow\left\{\begin{array}{l}P_{f}+f_{1}^{\curlyvee}+f_{3}^{x} \\ P_{f}+f_{1}^{x}+f_{2}^{\jmath}\end{array}\right\} \equiv P_{f}+$ visible + “invisible". Here either $f_{1}$ or $f_{2}$ gets detected, e.g., when $f_{1} f_{2}=\ell \nu_{\ell}$, $\ell \nu_{S}, \ell f^{\mathrm{DM}}, \ell f^{\mathrm{LLP}}$.

(S3) $P_{i} \rightarrow P_{f}+f_{1}^{x}+f_{2}^{x} \equiv P_{f}+$ invisible. Here neither $f_{1}$ nor $f_{2}$ gets detected, e.g., when $f_{1} f_{2}=\nu_{\ell} \bar{\nu}_{\ell}, \nu_{\ell} \bar{\nu}_{S}$, $\nu_{S} \bar{\nu}_{\ell}, \nu_{S} \bar{\nu}_{S}, f^{\mathrm{DM}} \bar{f}^{\mathrm{DM}}, f_{1}^{\mathrm{DM}} f_{2}^{\mathrm{DM}}, f_{1}^{\mathrm{LLP}} f_{2}^{\mathrm{LLP}}$, etc.

It should be noted that the above classification is based on our existing experimental explorations. What is undetected today might get detected in the future with advanced detectors. In such a case, we can imagine that, in the future, the modes grouped in S2 might migrate to S1, and those in S3 might be grouped under S2. Below we explore each of the above scenarios in more detail. 


\section{B. Exploration of new physics effects in each scenario}

The first scenario (S1) is an experimenter's delight, as in this case all final 4-momenta can be easily measured and the $t$-vs- $u$ Dalitz plot can be obtained. Here our methodology can be used to look for the possible signature of new physics in rare decays such as $B \rightarrow D \ell^{-} \ell^{+}$(which can be found in Ref. [10]) or to study the nature of new physics

$$
\frac{1}{\Gamma} \frac{d \Gamma}{d \cos \theta}=\left\{\begin{array}{l}
1 / 2 \\
T_{0}+T_{2} \cos ^{2} \theta \\
T_{0}+T_{1} \cos \theta+T_{2} \cos ^{2} \theta
\end{array}\right.
$$

where $T_{2}=3\left(1 / 2-T_{0}\right)$, with the quantities $T_{0}, T_{1}$, and $T_{2}$ being easily obtainable from the Dalitz plot distribution by using Eq. (18). It is clear from Eq. (19) that a scalar or pseudoscalar interaction would give rise to a uniform (or constant) angular distribution, while a tensorial interaction gives a nonuniform distribution which is symmetric under $\cos \theta \leftrightarrow-\cos \theta$ and for this $T_{0} \leq 1 / 2$. On the other hand, a vector or axial-vector interaction can be described only by the most general form of the angular distribution, with its signature being $T_{1} \neq 0$. Nevertheless, if a vector or axialvector interaction contributes to the flavor violating processes $B \rightarrow P \ell^{-} \ell^{\prime+}$, it is important to note that $T_{1} \propto\left(m_{\ell}^{2}-m_{\ell^{\prime}}^{2}\right)$, where $m_{\ell}, m_{\ell^{\prime}}$ denote the masses of the charged leptons $\ell^{-}$and $\ell^{\prime+}$, respectively. Therefore, we should observe an increase in the value of $T_{1}$ when going from $B \rightarrow P \mu^{-} e^{+}$to $B \rightarrow P \tau^{-} \mu^{+}$to $B \rightarrow P \tau^{-} e^{+}$. This would nail down the vector or axial-vector nature of the NP if it is the only NP contributing to these decays. Thus far we have analyzed the first scenario (S1) in which the relevant decays can be easily probed with existing detectors.

The second scenario (S2) can also be studied experimentally with existing detectors. In this case, the missing 4-momentum can be fully deduced using conservation of 4-momentum. Thus the $t$-vs- $u$ Dalitz plot can readily be obtained. Using our methodology, the signatures of NP can then be extracted. One promising candidate for search for $\mathrm{NP}$ in this kind of scenario is in the decay $B \rightarrow P \ell N$, where $P=\pi, K$, or $D$ and $N$ can be an active neutrino $\left(\nu_{\ell}\right)$ or sterile neutrino $\left(\nu_{S}\right)$ or a neutral dark fermion $\left(f^{\mathrm{DM}}\right)$ or a long-lived neutral fermion ( $\left.f^{\mathrm{LLP}}\right)$ which decays outside the detector. These S2 decay modes offer an exciting opportunity for the study of NP effects.

The third scenario (S3), which has the maximum number of NP possibilities, is also the most challenging one for the

\footnotetext{
${ }^{4}$ For some specific NP scenarios which can lead to such leptonflavor violating decays and for recent developments, see Ref. [16].
}

contributing to lepton-flavor violating processes such as $B \rightarrow P \ell^{ \pm} \ell^{\prime \mp}$, where $P=\pi, K, D, \ell \neq \ell^{\prime}$ and $\ell, \ell^{\prime}=e, \mu$, $\tau$. Let us consider a few NP possibilities mediating this lepton-flavor violating decay. There is no contribution within the SM to such decays. Therefore, all contributions to these decays come from NP alone. ${ }^{4}$ It is very easy to note that, for the decay $B \rightarrow P \ell^{-} \ell^{\prime+}$, from Eqs. (8) and (11) we get

(only scalar or pseudoscalar interaction),

(only tensorial interaction),

(only vector or axial-vector interaction),

current generation of experimental facilities due to the lack of information about the individual 4-momentum of $f_{1}$ and $f_{2}$. This implies that we cannot do any angular analysis for these kinds of decays unless by some technological advancement such as by using displaced vertex detectors ${ }^{5}$ we can manage to make measurement of the 4-momentum or the angular information of at least one of the final fermions. Getting 4-momenta of both of the fermions would be ideal, but knowing the 4-momentum of either one of them would suffice for our purposes. We are optimistic that the advancement in detector technology would push the current S3 decay modes to get labeled as S2 modes in the foreseeable future. It is important to note that, once the current S3 modes enter the S2 category, we can cover the whole spectrum of NP possibilities in the $P_{i} \rightarrow P_{f} f_{1} f_{2}$ decays. Below we make a comprehensive exploration of NP possibilities in the generalized S2 decay modes, which includes the current $\mathrm{S} 2$ and $\mathrm{S} 3$ modes together.

\section{Probing the effects of new physics in the $\mathbf{S 2}$ and generalized $\mathbf{S 2}$ scenarios}

In the generalized S2 (GS2) scenario, we have decays of the type

$$
P_{i} \rightarrow\left\{\begin{array}{l}
P_{f}+f_{1}^{\mathcal{}}+f_{2}^{x} \\
P_{f}+f_{1}^{x}+f_{2}^{\mathcal{V}}
\end{array}\right\} \equiv P_{f}+\text { visible }+ \text { invisible }
$$

where the detected $(\boldsymbol{})$ or undetected $(\boldsymbol{X})$ nature is not constrained by our existing detector technology. In some cases, even with advanced detectors, either of the fermions $f_{1}, f_{2}$ might not get detected simply because its direction of flight lies outside the finite detector coverage, especially

\footnotetext{
${ }^{5}$ There are many existing proposals for such displaced vertex studies from other theoretical and experimental considerations (see Refs. [17,18] and references contained therein for further information).
} 
when the detector is located farther from the place of origin of the particle. Such possibilities are also included here. As noted before, measuring the 4-momentum of either of the final fermions would suffice to carry out the angular analysis following our approach.

In this context, let us analyze the following decays.

(i) S2 decay: $B \rightarrow P \ell^{-} f^{x}$, where $P$ can be $\pi$ or $D$ and where $f^{x}$ is a neutral fermion. In the SM, this process is mediated by a $W^{-}$boson and we have $f^{x}=\bar{\nu}_{\ell}$. The presence of NP can imply $f^{x}$ being a sterile neutrino $\nu_{S}$ or a fermionic dark matter particle $f^{\mathrm{DM}}$ or a long-lived fermion $f^{\mathrm{LLP}}$, with additional non-SM interactions.

(ii) GS2 decay: $B \rightarrow K f_{1}^{\checkmark} f_{2}^{x}$ where $f_{1}^{\checkmark}$ and $f_{2}^{x}$ are both neutral fermions. In the SM, this process is mediated by a $Z^{0}$ boson and we have $f_{1} f_{2}=\nu_{\ell} \bar{\nu}_{\ell}$. However, in the case of NP contribution, we can get pairs of sterile neutrinos or fermionic dark matter or fermionic long-lived particles, etc., along with nonstandard interactions as well. Here we are assuming that either of the final neutral fermions leaves a displaced vertex signature in an advanced detector so that its 4-momentum or angular information could be obtained. If the reconstruction of $f_{1}^{\checkmark}$ is not precise enough to distinguish it from active neutrinos, then the angular distribution could be used to look for the signature of NP. We shall explicitly explore this interesting aspect in our analysis.

\section{New physics effects in the $S 2$ decay $B \rightarrow P \ell^{-} f^{x}$}

Analyzing the $B \rightarrow P \ell^{-} f^{x}$ decay in the SM, we find that only vector and axial vector currents contribute and that $F_{A}^{ \pm}=-F_{V}^{ \pm}$, while other form factors are zero. Also considering the antineutrino to be massless, i.e., $m_{2}=0$ we find that

$$
\begin{aligned}
a_{t} & =m_{\ell}^{2}+m_{P}^{2}+\left(s+m_{\ell}^{2}\right)\left(m_{B}^{2}-m_{P}^{2}-s\right) /(2 s), \\
a_{u} & =m_{P}^{2}+\left(s-m_{\ell}^{2}\right)\left(m_{B}^{2}-m_{P}^{2}-s\right) /(2 s), \\
b & =\left(s-m_{\ell}^{2}\right) \sqrt{\lambda\left(s, m_{B}^{2}, m_{P}^{2}\right)} /(2 s),
\end{aligned}
$$

where $m_{\ell}, m_{P}$, and $m_{B}$ denote the masses of the charged lepton $\ell^{-}$and mesons $P$ and $B$, respectively. Substituting this information into Eqs. (9) and (7), we get

$$
\frac{d^{2} \Gamma^{\mathrm{SM}}}{d s d \cos \theta}=\frac{b \sqrt{s}\left(C_{0}^{\mathrm{SM}}+C_{1}^{\mathrm{SM}} \cos \theta+C_{2}^{\mathrm{SM}} \cos ^{2} \theta\right)}{128 \pi^{3} m_{B}^{2}\left(m_{B}^{2}-m_{P}^{2}+s\right)},
$$

where

$$
\begin{aligned}
C_{0}^{\mathrm{SM}}= & 4\left(| ( F _ { V } ^ { + } ) _ { \mathrm { SM } } | ^ { 2 } \left(\lambda\left(s, m_{B}^{2}, m_{P}^{2}\right)-m_{\ell}^{2}\left(s-2\left(m_{B}^{2}-m_{P}^{2}\right)\right)\right.\right. \\
& \left.-m_{\ell}^{4}\left(m_{B}^{2}-m_{P}^{2}\right)^{2} / s^{2}\right)+\left|\left(F_{V}^{-}\right)_{\mathrm{SM}}\right|^{2} m_{\ell}^{2}\left(s-m_{\ell}^{2}\right) \\
& \left.+2 \operatorname{Re}\left(\left(F_{V}^{+}\right)_{\mathrm{SM}}\left(F_{V}^{-}\right)_{\mathrm{SM}}^{*}\right) m_{\ell}^{2}\left(m_{B}^{2}-m_{P}^{2}\right)\left(1-\frac{m_{\ell}^{2}}{s}\right)\right),
\end{aligned}
$$

$$
\begin{aligned}
C_{1}^{\mathrm{SM}}= & 16 m_{\ell}^{2} b\left(\left(\frac{m_{B}^{2}-m_{P}^{2}}{s}\right)\left|\left(F_{V}^{+}\right)_{\mathrm{SM}}\right|^{2}\right. \\
& \left.+\operatorname{Re}\left(\left(F_{V}^{+}\right)_{\mathrm{SM}}\left(F_{V}^{-}\right)_{\mathrm{SM}}^{*}\right)\right)
\end{aligned}
$$

$C_{2}^{\mathrm{SM}}=-16 b^{2}\left|\left(F_{V}^{+}\right)_{\mathrm{SM}}\right|^{2}$.

It is important to notice that in Eq. (21) we have many terms in the expression for $C_{0}^{\mathrm{SM}}$ that are proportional to some power of the lepton mass, while the entire $C_{1}^{\mathrm{SM}}$ is directly proportional to $m_{\ell}^{2}$. If we compare the $m_{\ell}$ dependent and $m_{\ell}$ independent contributions in $C_{0}^{\mathrm{SM}}$, we find that the dependent terms are suppressed by about a factor of $\mathcal{O}\left(2 m_{\ell}^{2} / m_{B}^{2}\right)$, which is roughly $8 \times 10^{-4}$ for a muon and $2 \times 10^{-8}$ for an electron. Thus we can neglect these $m_{\ell}$ dependent terms in comparison with mass independent terms. Equivalently, we can consider charged leptons such as electrons and muons as massless fermions, when compared with the $B$ meson mass scale. In the limit $m_{\ell} \rightarrow 0$, the expression for angular distribution given in Eq. (20) becomes much simpler,

$$
\frac{d^{2} \Gamma^{\mathrm{SM}}}{d s d \cos \theta}=\frac{b^{3} \sqrt{s}}{8 \pi^{3} m_{B}^{2}\left(m_{B}^{2}-m_{P}^{2}+s\right)}\left|\left(F_{V}^{+}\right)_{\mathrm{SM}}\right|^{2} \sin ^{2} \theta .
$$

Independent of the expression for $\left(F_{V}^{+}\right)_{\mathrm{SM}}$, equivalently independent of any hadronic uncertainties, it is easy to show that the normalized angular distribution in the SM is given by

$$
\frac{1}{\Gamma^{\mathrm{SM}}} \frac{d \Gamma^{\mathrm{SM}}}{d \cos \theta}=\frac{3}{4} \sin ^{2} \theta
$$

which implies that $T_{0}=3 / 4=-T_{2}, T_{1}=0$. Since the distribution of events in the Dalitz plot is symmetric under $\cos \theta \leftrightarrow-\cos \theta$, we have $N_{\text {I }}=N_{\text {IV }}$ and $N_{\text {II }}=N_{\text {III }}$, which automatically satisfies the condition $T_{1}=0$. If we solve $T_{0}=3 / 4=-T_{2}$, we find that the number of events in the different segments of the Dalitz plot (equivalently, the number of events in the four distinct bins of $\cos \theta$ ) are related to one another by

$$
\frac{N_{\mathrm{I}}}{N_{\mathrm{II}}}=\frac{5}{11}=\frac{N_{\mathrm{IV}}}{N_{\mathrm{III}}} .
$$


Any significant deviation from this would imply the presence of NP effects. To illustrate the effects of NP on the angular distribution in these types of decays, we consider two simple and specific NP possibilities. Here we assume the charged lepton to be massless $\left(m_{\ell}=0\right)$ and the undetected fermion $\left(f^{x}\right)$ to have mass $m \neq 0$.

Scalar-type new physics. - Considering the simplest scalartype NP scenario, with $F_{S} \neq 0, F_{P}=F_{V}^{ \pm}=F_{A}^{ \pm}=F_{T_{1}}=$ $F_{T_{2}}=0$, we get $C_{0}^{\mathrm{NP}}=2\left(s-m^{2}\right)\left|F_{S}\right|^{2}$ and $C_{1}^{\mathrm{NP}}=0=C_{2}^{\mathrm{NP}}$. In other words, there is no angular dependence at all here, i.e.,

$$
\frac{d^{2} \Gamma^{\mathrm{NP}}}{d s d \cos \theta}=\frac{b \sqrt{s}}{64 \pi^{3} m_{B}^{2}\left(m_{B}^{2}-m_{P}^{2}+s\right)}\left(s-m^{2}\right)\left|F_{S}\right|^{2},
$$

where $b=\frac{s-m^{2}}{2 s} \sqrt{\lambda\left(s, m_{B}^{2}, m_{P}^{2}\right)}$ and $m^{2} \leq s \leq\left(m_{B}-m_{P}\right)^{2}$. If we do the integration over $s$, then the normalized angular distribution, independent of any hadronic uncertainties, is constant and equals $1 / 2$. In fact, if such a new physics were present, our observation of $B \rightarrow P+\ell^{-}+f^{x}$ would have the following angular distribution,

$$
\frac{d \Gamma}{d \cos \theta}=\Gamma^{\mathrm{SM}}\left(\frac{3}{4} \sin ^{2} \theta+\frac{1}{2} \epsilon_{0}\right),
$$

where we have parametrized the new physics contribution in terms of $\epsilon_{0}=\Gamma^{\mathrm{NP}} / \Gamma^{\mathrm{SM}}$. Here $\epsilon_{0}$ is not independent of any hadronic uncertainties since, in the evaluation of both $\Gamma^{\mathrm{SM}}$ and $\Gamma^{\mathrm{NP}}$, the respective form factors play a major role. Nevertheless, $\epsilon_{0}$ is an effective parameter that measures how large or small the NP contribution is with respect to that of the SM. Doing integration over $\cos \theta$, we get $\Gamma=\Gamma^{\mathrm{SM}}\left(1+\epsilon_{0}\right)=\Gamma^{\mathrm{SM}}+\Gamma^{\mathrm{NP}}$. This implies

$$
\frac{1}{\Gamma} \frac{d \Gamma}{d \cos \theta}=\frac{3 \sin ^{2} \theta+2 \epsilon_{0}}{4\left(1+\epsilon_{0}\right)} .
$$

This angular distribution is shown in Fig. 4, where we have varied $\epsilon_{0}$ in the range $[0,1]$, i.e., we have allowed for the possibility that the NP contribution might be as large as that of the SM. It is interesting to find that in Fig. 4, at two specific values of $\cos \theta$, there is no difference between the standard model prediction alone and the combination of standard model and new physics contributions. These two points can be easily obtained by equating Eqs. (23) and (25), and then solving for $\cos \theta$ gives us

$$
\cos \theta= \pm 1 / \sqrt{3} \approx \pm 0.57735
$$

This corresponds to the angle $\theta \approx 54.74^{\circ}$. At these two points in $\cos \theta$, the normalized uniangular distribution always has the value 0.5 , even if there is some scalar new physics contributing to our process under consideration.

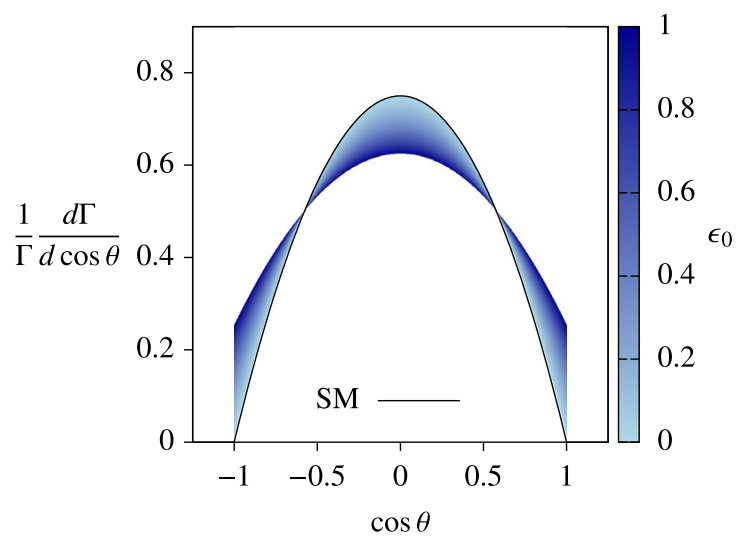

FIG. 4. Normalized uniangular distribution showing the effect of a scalar new physics contribution to $B \rightarrow P \ell^{-} f^{x}$ where we have neglected the mass of the charged lepton $\ell=e, \mu$. This also shows the normalized uniangular distribution showing the effect of a scalar new physics contribution to $B \rightarrow K f_{1}^{\downarrow} f_{2}^{x}$ considering the $m_{1}=m_{2}$ case only.

From Eq. (25), it is clear that, despite the scalar NP effect, the distribution is still symmetric under $\cos \theta \leftrightarrow-\cos \theta$, and solving for the number of events in the four segments of the Dalitz plot (equivalently, the four $\cos \theta$ bins), we get

$$
\frac{N_{\mathrm{I}}}{N_{\mathrm{II}}}=\frac{5+8 \epsilon_{0}}{11+8 \epsilon_{0}}=\frac{N_{\mathrm{IV}}}{N_{\mathrm{III}}} .
$$

It is easy to see that when $\epsilon_{0}=0$ we get back the SM prediction of Eq. (24) as expected.

Tensor-type new physics. - Let us consider a tensor type of new physics possibility in which $F_{T_{1}} \neq 0$ and in which all other form factors are zero. In such a case, we get

$$
\begin{aligned}
& C_{0}^{\mathrm{NP}}=2 m^{2}\left(s-m^{2}\right) \frac{\lambda\left(s, m_{B}^{2}, m_{P}^{2}\right)}{s}\left|F_{T_{1}}\right|^{2}, \\
& C_{1}^{\mathrm{NP}}=0, \\
& C_{2}^{\mathrm{NP}}=2\left(s-m^{2}\right)^{2} \frac{\lambda\left(s, m_{B}^{2}, m_{P}^{2}\right)}{s}\left|F_{T_{1}}\right|^{2} .
\end{aligned}
$$

It is easy to notice that in the limit $m \rightarrow 0$ we have $C_{0} \rightarrow 0$ but $C_{2} / \rightarrow 0$. If we do the integration over $s$, then the normalized angular distribution is given by

$$
\frac{1}{\Gamma^{\mathrm{NP}}} \frac{d \Gamma^{\mathrm{NP}}}{d \cos \theta}=T_{0}^{\mathrm{NP}}+T_{2}^{\mathrm{NP}} \cos ^{2} \theta,
$$

where $T_{2}^{\mathrm{NP}}=3\left(1 / 2-T_{0}^{\mathrm{NP}}\right)$ and $T_{0}^{\mathrm{NP}}=3 c_{0} /\left(6 c_{0}+2 c_{2}\right)$ with

$$
c_{j}=\int_{m^{2}}^{\left(m_{B}-m_{P}\right)^{2}} \frac{b \sqrt{s} C_{j}^{\mathrm{NP}}}{128 \pi^{3} m_{B}^{2}\left(m_{B}^{2}-m_{P}^{2}+s\right)} d s .
$$


Here, by construction itself, the $T_{0}^{\mathrm{NP}}$ and $T_{2}^{\mathrm{NP}}$ terms are independent of any form factors or, equivalently, free from any hadronic uncertainties. It is also easy to notice that in the limit $m \rightarrow 0$ we have $T_{0}=0$. If such a new physics were present, our observation of $B \rightarrow P \ell^{-} f^{x}$ would have the following angular distribution,

$\frac{d \Gamma}{d \cos \theta}=\Gamma^{\mathrm{SM}}\left(\frac{3}{4} \sin ^{2} \theta+\left(T_{0}^{\mathrm{NP}}+3\left(\frac{1}{2}-T_{0}^{\mathrm{NP}}\right) \cos ^{2} \theta\right) \epsilon\right)$,

where $\epsilon=\Gamma^{\mathrm{NP}} / \Gamma^{\mathrm{SM}}$ is the NP parameter which can vary in the range $[0,1]$, denoting the possibility that the NP contribution can be as large as that of the $\mathrm{SM}$, and $T_{0}^{\mathrm{NP}}$ acts as a free parameter here which can vary in the range $[0,3 / 4]$, in which $d \Gamma^{\mathrm{NP}} / d \cos \theta \geq 0$ for all values of $\cos \theta$. It must be noted here that $\epsilon$ does depend on the appropriate form factors, and hence it has hadronic uncertainties. Doing an integration over $\cos \theta$, we get $\Gamma=\Gamma^{\mathrm{SM}}(1+\epsilon)=\Gamma^{\mathrm{SM}}+\Gamma^{\mathrm{NP}}$. This implies

$$
\frac{1}{\Gamma} \frac{d \Gamma}{d \cos \theta}=\frac{3+4 T_{0}^{\mathrm{NP}} \epsilon-3\left(4 T_{0}^{\mathrm{NP}} \epsilon-2 \epsilon+1\right) \cos ^{2} \theta}{4(1+\epsilon)} .
$$

This angular distribution is shown in Fig. 5, in which we have considered nine values of $T_{0}^{\mathrm{NP}}$ and varied $\epsilon$ in the range $[0,1]$. It is clearly evident in Fig. 5 that the $T_{0}^{\mathrm{NP}}=$ $3 / 4$ case is always indistinguishable from the SM case, as it should be. Just like the scalar-type new physics case, we observe that there are two values of $\cos \theta$ at which there is no difference between the SM prediction alone and the

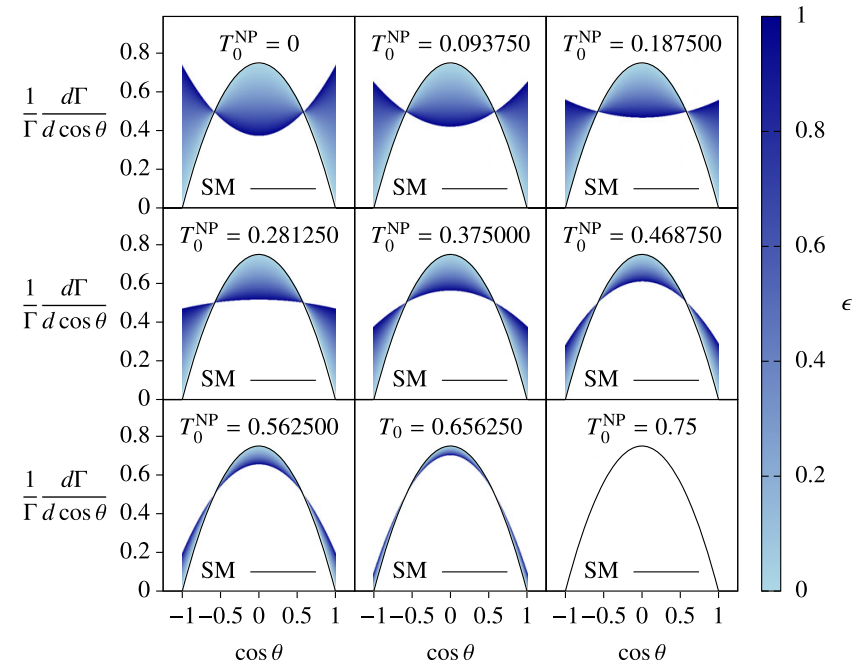

FIG. 5. Normalized uniangular distribution showing the effect of a tensor new physics contribution to $B \rightarrow P \ell^{-} f^{x}$, where we have neglected the mass of the charged lepton $\ell=e, \mu$. This set of plots can also describe the effect of a vector new physics contribution to $B \rightarrow K f_{1}^{J} f_{2}^{x}$ when the final fermions are equally massive. combination of SM and NP contributions. These two points can be easily computed by equating Eqs. (23) and (30), and then solving for $\cos \theta$, we once again find that

$$
\cos \theta= \pm 1 / \sqrt{3} \approx \pm 0.57735,
$$

which corresponds to the angle $\theta \approx 54.74^{\circ}$. At these two points in $\cos \theta$, the normalized uniangular distribution always has the value 0.5 , even if there is some tensor new physics contributing to our process under consideration. It should be noted that these are also the same points where the scalar new physics contribution shows a similar effect.

It is also easy to notice that the angular distribution given in Eq. (30) is symmetric under $\cos \theta \leftrightarrow-\cos \theta$, and solving for the number of events in the four segments of the Dalitz plot (equivalently, the four $\cos \theta$ bins), we get

$$
\frac{N_{\mathrm{I}}}{N_{\mathrm{II}}}=\frac{5+2 \epsilon\left(7-6 T_{0}^{\mathrm{NP}}\right)}{11+2 \epsilon\left(1+6 T_{0}^{\mathrm{NP}}\right)}=\frac{N_{\mathrm{IV}}}{N_{\mathrm{III}}} .
$$

It is easy to see that, when $\epsilon=0$ or $T_{0}^{\mathrm{NP}}=3 / 4$, we get back the SM prediction of Eq. (24), as expected.

Finally, we analyze new physics possibilities in the decays belonging to the GS2 category. Because of the very nature of the GS2 decay modes, the following discussion of NP effects presumes the usage of advanced detector technology to get angular information.

\section{New physics effects in the GS2 decay $B \rightarrow K f_{1}^{\checkmark} f_{2}^{X}$}

As mentioned before, the GS2 decay modes were originally part of S3, i.e., it is extremely difficult to get angular distribution for these cases unless we innovate on detector technology. Here we consider such a decay mode, $B \rightarrow K f_{1}^{\mathcal{J}} f_{2}^{x}$, in which both $f_{1}, f_{2}$ are neutral fermions who have evaded, till now, all of our attempts to detect them near their place of origin. But probably, with displaced vertex detectors or some other advanced detector, we could bring at least one of these fermions (say, $f_{1}$ ) under the purview of experimental study and measure its 4-momentum or angular information. The missing fermion (which is $f_{2}$ in our example here) might have flown in a direction along which there is no detector coverage. To increase the sample size, we should include $B \rightarrow K f_{1}^{x} f_{2}^{v}$ events also provided that we know how to ascertain the particle or antiparticle nature of $f_{1}$ and $f_{2}$. To illustrate this point, let us consider the possibility $f_{1} f_{2}=\nu_{S} \bar{\nu}_{S}$. In a displaced vertex detector, if we see $\pi^{+} \mu^{-}$events, they can be attributed to the decay of $\nu_{S}$, and similarly $\pi^{-} \mu^{+}$events would appear from the decay of $\bar{\nu}_{S}$. In this case, we can infer the angle $\theta$ by knowing the 4-momentum of either $f_{1}=\nu_{S}$ or $f_{2}=\bar{\nu}_{S}$ (see Fig. 2). If we find that both $f_{1}$ and $f_{2}$ leave behind their signature tracks in the detector (i.e., $f_{1}^{\checkmark} f_{2}^{\mathcal{}}$ ) it would be the most ideal situation. But as we have already stressed, measuring the 
4-momenta of either of the fermions would suffice for our angular studies.

In the SM, the only contribution to $B \rightarrow K f_{1}^{\mathcal{J}} f_{2}^{x}$ and $B \rightarrow K f_{1}^{x} f_{2}^{\checkmark}$ would come from $B \rightarrow K \nu_{\ell} \bar{\nu}_{\ell}$, where, as in the case of NP, we have a number of possibilities that includes sterile neutrinos, dark matter particles, and some long-lived particles in the final state, $f_{1} f_{2}=\nu_{\ell} \bar{\nu}_{S}, \nu_{S} \bar{\nu}_{\ell}$, $\nu_{S} \bar{\nu}_{S}, f^{\mathrm{DM}} \bar{f}^{\mathrm{DM}}, f_{1}^{\mathrm{DM}} f_{2}^{\mathrm{DM}}, f^{\mathrm{LLP}} \bar{f}^{\mathrm{LLP}}, f_{1}^{\mathrm{LLP}} f_{2}^{\mathrm{LLP}}$, etc. ${ }^{6}$ One can also consider nonstandard neutrino interactions also contributing in these cases. To demonstrate our methodology, we shall analyze only a subset of these various NP possibilities in which $f_{1}$ and $f_{2}$ have the same mass, i.e., $m_{1}=m_{2}=m$ (say), as this greatly simplifies the calculation. As we shall illustrate below, we not only can detect the presence of NP but can ascertain whether it is of scalar type or vector type, e.g., by analyzing the angular distribution.

Before we go for new physics contributions, let us analyze the SM contribution $B \rightarrow K \nu_{\ell} \bar{\nu}_{\ell}$. Here there are only vector and axial-vector currents contributions, and $F_{A}^{ \pm}=-F_{V}^{ \pm}$. Also the neutrino and antineutrino are massless, i.e., $m_{1}=0=m_{2}$, which implies $a_{t}=a_{u}=$ $\frac{1}{2}\left(m_{B}^{2}+m_{K}^{2}-s\right)$ and $b=\frac{1}{2} \sqrt{\lambda\left(s, m_{B}^{2}, m_{K}^{2}\right)}$, where $m_{B}$ and $m_{K}$ denote the masses of $B$ and $K$ mesons, respectively. Substituting this information into Eqs. (9) and (7), we get the expression for $\frac{d^{2} \Gamma^{\mathrm{SM}}}{d s d \cos \theta}$ that is identical to Eq. (22) with the appropriate substitution $m_{P} \rightarrow m_{K}$. Irrespective of the expression for the form factor, i.e., independent of any hadronic uncertainties, it can be easily shown that the normalized angular distribution in the SM is then given by Eq. (23). Following the same logic as that given after Eq. (23), we find that the number of events in the different segments of the Dalitz plot (equivalently, the number of events in the four distinct bins of $\cos \theta$ ) are related to one another by Eq. (24). This sets the stage for us to explore (i) a scalar type and (ii) a vector type of NP possibility, with final fermions for which $m_{1}=m_{2}=m \neq 0$.

Scalar-type new physics.-Once again we consider the simplest scalar-type NP scenario, with $F_{S} \neq 0$, and other form factors being zero. This leads us to

$$
\begin{aligned}
& C_{0}^{\mathrm{NP}}=2\left(s-4 m^{2}\right)\left|F_{S}\right|^{2}, \\
& C_{1}^{\mathrm{NP}}=0=C_{2}^{\mathrm{NP}} .
\end{aligned}
$$

In other words, there is no angular dependence at all here, i.e.,

\footnotetext{
${ }^{6}$ In addition to the new physics possibilities considered here, there can be additional contributions to the $B \rightarrow K+$ invisible decay, e.g., from SM singlet scalars contributing to the invisible part discussed in Ref. [19]. As is evident, our analysis is instead focused on a pair of fermions contributing to the invisible part.
}

$\frac{d^{2} \Gamma^{\mathrm{NP}}}{d s d \cos \theta}=\frac{b \sqrt{s}}{64 \pi^{3} m_{B}^{2}\left(m_{B}^{2}-m_{K}^{2}+s\right)}\left(s-4 m^{2}\right)\left|F_{S}\right|^{2}$,

where $b=\sqrt{\left(s-4 m^{2}\right) \lambda\left(s, m_{B}^{2}, m_{K}^{2}\right)} /(2 \sqrt{s})$ and $4 m^{2} \leq s \leq$ $\left(m_{B}-m_{K}\right)^{2}$. If we do the integration over $s$, then for NP only the normalized angular distribution, without any hadronic uncertainties, is $1 / 2$ at all values of $\cos \theta$. Considering such a NP contributing in addition to the $\mathrm{SM}$, the experimentally observed angular distribution can be written as

$$
\frac{d \Gamma}{d \cos \theta}=\Gamma^{\mathrm{SM}}\left(\frac{3}{4} \sin ^{2} \theta+\frac{1}{2} \epsilon_{0}\right),
$$

where $\epsilon_{0}=\Gamma^{\mathrm{NP}} / \Gamma^{\mathrm{SM}}$ is the new physics parameter which can vary in the range $[0,1]$ if we assume the NP contribution to be as large as that from the SM. Once again $\epsilon_{0}$ does depend on the appropriate form factors. Doing an integration over $\cos \theta$, we get $\Gamma=\Gamma^{\mathrm{SM}}\left(1+\epsilon_{0}\right)=$ $\Gamma^{\mathrm{SM}}+\Gamma^{\mathrm{NP}}$. This implies

$$
\frac{1}{\Gamma} \frac{d \Gamma}{d \cos \theta}=\frac{3 \sin ^{2} \theta+2 \epsilon_{0}}{4\left(1+\epsilon_{0}\right)} .
$$

Since Eq. (34) is identical to Eq. (25), the angular distribution for this case is also as shown in Fig. 4, where we have varied $\epsilon_{0}$ in the range $[0,1]$. Once again at two specific values of $\cos \theta$-namely, $\cos \theta= \pm 1 / \sqrt{3} \approx$ \pm 0.57735 corresponding to the angle $\theta \approx 54.74^{\circ}$ - there is no difference between the standard model prediction alone and the combination of the standard model and scalar new physics contributions. At these two points in $\cos \theta$, the normalized uniangular distribution always has the value 0.5 , even if there is some scalar new physics contributing to our process under consideration.

Since the angular distribution shown in Eq. (34) is fully symmetric under $\cos \theta \leftrightarrow-\cos \theta$, the number of events in the four segments of the Dalitz plot (equivalently, in the four $\cos \theta$ bins) satisfies the following relationship,

$$
\frac{N_{\mathrm{I}}}{N_{\text {II }}}=\frac{5+8 \epsilon_{0}}{11+8 \epsilon_{0}}=\frac{N_{\text {IV }}}{N_{\text {III }}} .
$$

It is easy to see that $\epsilon_{0}=0$ gives the SM prediction of 5/11, as expected.

Vector-type new physics.-Let us now discuss another new physics scenario, such as the case of a flavor-changing $Z^{\prime}$ or a dark photon $\gamma_{D}$ giving rise to the final pair of fermions, $f_{1} f_{2}$. We assume that, for this kind of new physics scenario, $F_{V}^{+}=F_{V}^{\mathrm{NP}} \neq 0$, and other form factors are zero. ${ }^{7}$ For this kind of new physics, we get

\footnotetext{
${ }^{7}$ We can even consider $F_{V}^{-} \neq 0$ here. However, it is clear from Eq. (8) that the additional terms containing $F_{V}^{-}$vanish identically when we consider $m_{1}=m_{2}=m \neq 0$.
} 


$$
\begin{aligned}
& C_{0}^{\mathrm{NP}}=2\left|F_{V}^{\mathrm{NP}}\right|^{2} \lambda\left(s, m_{B}^{2}, m_{K}^{2}\right), \\
& C_{1}^{\mathrm{NP}}=0, \\
& C_{2}^{\mathrm{NP}}=-8 b^{2}\left|F_{V}^{\mathrm{NP}}\right|^{2} .
\end{aligned}
$$

The angular distribution for the NP alone contribution can, therefore, be written in terms of $T_{0}^{\mathrm{NP}}$ and $T_{2}^{\mathrm{NP}}$, which are directly proportional to $C_{0}^{\mathrm{NP}}$ and $C_{2}^{\mathrm{NP}}$, respectively. It would lead us to describe the complete angular distribution in terms of $T_{0}^{\mathrm{NP}}$ and $\epsilon=\Gamma^{\mathrm{NP}} / \Gamma^{\mathrm{SM}}$ using Eq. (30), and the angular distribution would look like the one shown in Fig. 5. However, it is possible to describe the effects of NP on the angular distribution using a different set of parameters as well. For this, we start afresh with the angular distribution for the NP contribution alone, which in our case is given by

$$
\frac{d^{2} \Gamma^{\mathrm{NP}}}{d s d \cos \theta}=\frac{b\left|F_{V}^{\mathrm{NP}}\right|^{2} \lambda\left(s, m_{B}^{2}, m_{K}^{2}\right)\left(s \sin ^{2} \theta+4 m^{2} \cos ^{2} \theta\right)}{64 \pi^{3} m_{B}^{2}\left(m_{B}^{2}-m_{K}^{2}+s\right) \sqrt{s}} .
$$

Doing integration over $\cos \theta$, we obtain

$$
\frac{d \Gamma^{\mathrm{NP}}}{d s}=\frac{b\left|F_{V}^{\mathrm{NP}}\right|^{2} \lambda\left(s, m_{B}^{2}, m_{K}^{2}\right)}{64 \pi^{3} m_{B}^{2}\left(m_{B}^{2}-m_{K}^{2}+s\right) \sqrt{s}}\left(\frac{4 s+8 m^{2}}{3}\right) .
$$

Therefore, the normalized uniangular distribution, without any hadronic uncertainties, is given by

$$
\frac{1}{d \Gamma^{\mathrm{NP}} / d s} \frac{d^{2} \Gamma^{\mathrm{NP}}}{d s d \cos \theta}=\frac{3}{4}\left(\frac{s \sin ^{2} \theta+4 m^{2} \cos ^{2} \theta}{s+2 m^{2}}\right) .
$$

It is interesting to compare this with the standard model expression,

$$
\frac{1}{d \Gamma^{\mathrm{SM}} / d s} \frac{d^{2} \Gamma^{\mathrm{SM}}}{d s d \cos \theta}=\frac{3}{4} \sin ^{2} \theta .
$$

Since the range for $s$ is different in the SM and NP scenarios, we cannot add Eqs. (36) and (37) directly. Carrying out the integration over $s$, we get

$$
\frac{d \Gamma^{\mathrm{NP}}}{d \cos \theta}=\frac{3}{4}\left(\mathcal{S} \sin ^{2} \theta+\mathcal{C} \cos ^{2} \theta\right),
$$

where

$$
\begin{aligned}
& \mathcal{S}=\int_{4 m^{2}}^{\left(m_{B}-m_{K}\right)^{2}} \frac{d \Gamma^{\mathrm{NP}}}{d s}\left(\frac{s}{s+2 m^{2}}\right) d s, \\
& \mathcal{C}=\int_{4 m^{2}}^{\left(m_{B}-m_{K}\right)^{2}} \frac{d \Gamma^{\mathrm{NP}}}{d s}\left(\frac{4 m^{2}}{s+2 m^{2}}\right) d s .
\end{aligned}
$$

Doing an integration over $\cos \theta$, we get $\Gamma^{\mathrm{NP}}=\mathcal{S}+\mathcal{C} / 2$, and hence

$$
\frac{1}{\Gamma^{\mathrm{NP}}} \frac{d \Gamma^{\mathrm{NP}}}{d \cos \theta}=\frac{3\left(\mathcal{S} \sin ^{2} \theta+\mathcal{C} \cos ^{2} \theta\right)}{2(2 \mathcal{S}+\mathcal{C})} .
$$

Considering both the SM and NP contributions, the uniangular distribution for the process $B \rightarrow K f_{1}^{\mathcal{}} f_{2}^{x}$ is given by

$$
\frac{d \Gamma}{d \cos \theta}=\frac{3}{4} \Gamma^{\mathrm{SM}}\left(\left(1+\epsilon_{s}\right) \sin ^{2} \theta+\epsilon_{c} \cos ^{2} \theta\right),
$$

where $\epsilon_{s}=\mathcal{S} / \Gamma^{\mathrm{SM}}$ and $\epsilon_{c}=\mathcal{C} / \Gamma^{\mathrm{SM}}$ are the two parameters which describe the effect of vector-type NP. Thus the normalized angular distribution is given by

$$
\frac{1}{\Gamma} \frac{d \Gamma}{d \cos \theta}=\frac{3\left(1+\epsilon_{s}\right) \sin ^{2} \theta+3 \epsilon_{c} \cos ^{2} \theta}{4\left(1+\epsilon_{s}\right)+2 \epsilon_{c}} .
$$

It is important to note that $\epsilon_{c}$ and $\epsilon_{s}$ are dependent on the appropriate form factors and hence have hadronic uncertainties. It is also important to observe that, if we consider the mass of the fermion $f$ to be zero, i.e., $m=0$, then $\epsilon_{c}=0$ since $\mathcal{C}=0$. In such a case, the uniangular distribution is the same as that in the SM case. This is plausible, as in the SM case also, one has $m=0$ for the neutrino mass, and only vector and axial-vector currents contribute.

Assuming that the NP contribution can be smaller than or as large as the $\mathrm{SM}$ contribution, i.e., $0 \leq \Gamma^{\mathrm{NP}} \leq \Gamma^{\mathrm{SM}}$, we get

$$
0 \leq \epsilon_{s}+\epsilon_{c} / 2 \leq 1
$$

Thus $0 \leq \epsilon_{s} \leq 1$ implies that $0 \leq \epsilon_{c} \leq 2\left(1-\epsilon_{s}\right)$.

In Fig. 6, we have considered nine values of $\epsilon_{s}$ and have varied $\epsilon_{c}$ in the range $\left[0,2\left(1-\epsilon_{s}\right)\right]$ to obtain the uniangular distribution. It is clearly evident in Fig. 6 that the $\epsilon_{c}=0$

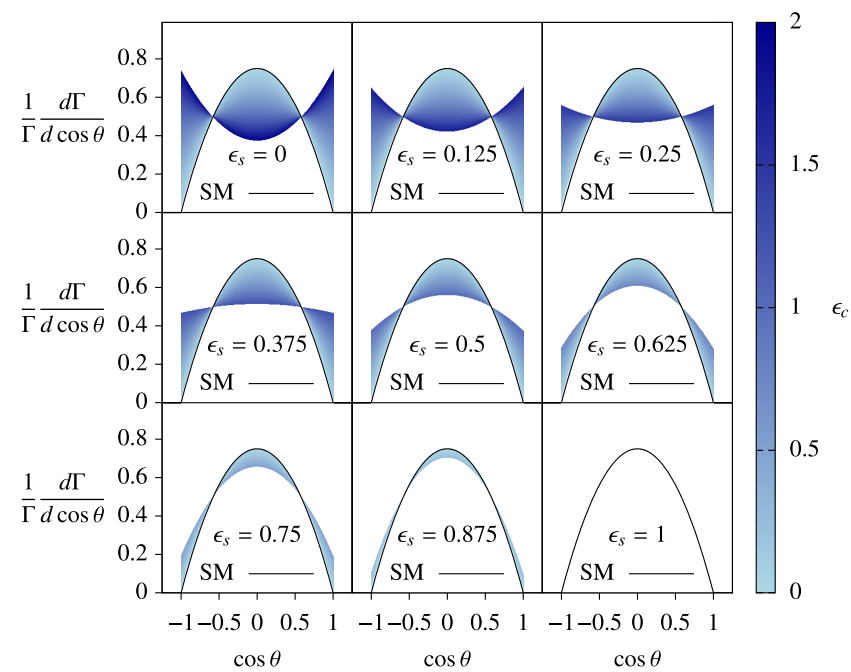

FIG. 6. Normalized uniangular distribution showing the effect of a vector new physics contribution to $B \rightarrow K f_{1}^{\checkmark} f_{2}^{x}$. 
case is always indistinguishable from the SM case, as it should be. Just like the scalar-type new physics case, we observe that, at $\cos \theta= \pm 1 / \sqrt{3} \approx \pm 0.57735$, there is no difference between the SM prediction alone and the combination of SM and NP contributions.

It is also easy to notice that the angular distribution given in Eq. (38) is symmetric under $\cos \theta \leftrightarrow-\cos \theta$, and solving for the number of events in the four segments of the Dalitz plot (equivalently, the four $\cos \theta$ bins), we get

$$
\frac{N_{\mathrm{I}}}{N_{\mathrm{II}}}=\frac{5\left(1+\epsilon_{s}\right)+7 \epsilon_{c}}{11\left(1+\epsilon_{s}\right)+\epsilon_{c}}=\frac{N_{\mathrm{IV}}}{N_{\mathrm{III}}} .
$$

It is easy to see that when $\epsilon_{c}=0=\epsilon_{s}$ we get back the SM prediction of $5 / 11$, as expected.

\section{Discussion}

It should be noted that our discussions on the types of NP contributions to the S2 and GS2 modes, specifically $B \rightarrow P \ell^{-} f^{x}$ and $B \rightarrow K f_{1}^{J} f_{2}^{X}$, respectively, has been fully general. There are no complications arising out of hadronic form factors since we have considered a normalized angular distribution. It should be noted that our analysis also does not depend on how large or small the masses of the fermions $f, f_{1,2}$ are, as long as they are nonzero.

It is also very interesting to note that both the scalar and tensor type of NP for the $B \rightarrow P \ell^{-} f^{x}$ decays, and both the scalar and vector types of NP for the $B \rightarrow K f_{1}^{\mathcal{J}} f_{2}^{x}$ decays, exhibit similar behaviors at $\cos \theta= \pm 1 / \sqrt{3}$. In order to know the real reason behind this, we must do a very general analysis. Let us assume that the most general angular distribution for the processes $B \rightarrow P \ell^{-} f^{x}$ and $B \rightarrow K f_{1}^{J} f_{2}^{x}$ is given by Eq. (11). If we now equate this distribution to the SM prediction of Eq. (23) and solve for $\cos \theta$ after substituting in Eq. (12), we find that

$$
\cos \theta=\frac{-c_{1} \pm \sqrt{c_{1}^{2}+3\left(c_{0}+c_{2}\right)^{2}}}{3\left(c_{0}+c_{2}\right)},
$$

where the $c_{j}$ 's (for $j=0,1,2$ ) are obtained from Eq. (13) with appropriate substitutions of masses and form factors. Thus Eq. (40) is the most general solution that we can get for the two specific values of $\cos \theta$. However, let us look at the specific case in which $c_{1}=0$. Only in this situation do we get $\cos \theta= \pm 1 / \sqrt{3}$. Now it is clear that since, in both the scalar and tensor types of NP considerations for the $B \rightarrow P \ell^{-} f^{x}$ decays and in both the scalar and vector types of NP considerations for the $B \rightarrow K f_{1}^{\mathcal{J}} f_{2}^{x}$ decays, the angular distribution did not have any term directly proportional to $\cos \theta$ (i.e., $c_{1}=0$ ), we obtained the same $\cos \theta=$ $\pm 1 / \sqrt{3}$ result in both the cases. Therefore, if the observed normalized uniangular distribution does not have the value 0.5 at $\cos \theta= \pm 1 / \sqrt{3}$, it implies that $c_{1} \neq 0$.
Another interesting aspect of the two specific NP contributions that we have considered is that, from Figs. 4-6, one can clearly see that the vector and tensor types of NP can accommodate a much larger variation in the angular distribution than the scalar-type NP. However, there is also a certain part of the angular distribution for which both scalar and vector (or tensor) types of NP give identical results. This happens when

$$
\epsilon_{0}=\frac{3 \epsilon_{c}}{2\left(1+\epsilon_{s}-\epsilon_{c}\right)}=\frac{\epsilon\left(3-4 T_{0}^{\mathrm{NP}}\right)}{1-\epsilon\left(2-4 T_{0}^{\mathrm{NP}}\right)} .
$$

In order for $\epsilon_{0}$ to vary in the range [0,1], we find that (i) for $0 \leq \epsilon_{s} \leq 1$, we have $0 \leq \epsilon_{c} \leq 2\left(1+\epsilon_{s}\right) / 5$ and (ii) for $0 \leq \epsilon \leq 1$, we have $\frac{1}{2} \leq T_{0}^{\mathrm{NP}} \leq \frac{3}{4}$. In these specific regions, therefore, it would not be possible to clearly distinguish whether scalar or vector- or tensor-type NP is contributing to our process under consideration. Nevertheless, our approach provides a methodology to constrain these specific NP hypotheses in a manner which does not require one to know the form factors or hadronic uncertainties a priori. The various $\epsilon$ 's we have introduced, though dependent on form factors, can effectively probe NP in a clean manner.

\section{CONCLUSION}

We have shown that all NP contributions to three-body semihadronic decays of the type $P_{i} \rightarrow P_{f} f_{1} f_{2}$, where $P_{i(f)}$ denotes an appropriate initial (final) pseudoscalar meson and $f_{1,2}$ are a pair of fermions, can be codified into the most general Lagrangian which gives rise to a very general angular distribution. The relevant NP information can be obtained by using various angular asymmetries, provided that at least one of the final pair of fermions has some detectable signature, such as a displaced vertex, in the detector. Depending on the detection feasibility of the final fermions, we have grouped the $P_{i} \rightarrow P_{f} f_{1} f_{2}$ decays into three distinct categories: (i) S1, where both $f_{1}$ and $f_{2}$ are detected, (ii) $\mathrm{S} 2$, where either $f_{1}$ or $f_{2}$ gets detected, and (ii) $\mathrm{S} 3$, where neither $f_{1}$ nor $f_{2}$ gets detected. We consider the possibility that, with advancement in detector technology, S3 decays could, in the future, be grouped under the S2 category. We analyze some specific NP scenarios in each of these categories to illustrate how NP affects the angular distribution. Specifically we have analyzed (a) lepton-flavor violating $\mathrm{S} 1$ decay $B \rightarrow P \ell^{-} \ell^{\prime+}$ (with $P=\pi, K$, $D$ and $\left.\ell, \ell^{\prime}=e, \mu, \tau\right)$ showing angular signatures of all generic NP possibilities, (b) S2 decays of the type $B \rightarrow$ $\mathrm{P}^{-} f$ (where $f$ is not detected in the laboratory) showing the effect of a scalar-type and a tensor-type NP on the angular distribution, and finally (c) S3 decays (more correctly, generalized S2 decays) of the type $B \rightarrow K f \bar{f}$ (where either $f$ or $\bar{f}$ gets detected in an advanced detector) showing the effects of a scalar-type and a vector-type NP on the angular distribution. The effects on the angular 
distribution can be easily estimated from Dalitz plot asymmetries. The signatures of NP in an angular distribution are distinct once the process is chosen carefully. Moreover, as shown in our examples, it can be possible, in certain cases, to do the identification and quantification of NP effects without worrying about hadronic uncertainties. We are optimistic that our methodology can be put to use in LHCb and Belle II in the study of appropriate $B$ meson decays, furthering our search for NP.

\section{ACKNOWLEDGMENTS}

This work was supported in part by the National Research Foundation of Korea (NRF) grant funded by the Korean Government (MSIP) (Grant No. NRF2018R1A4A1025334). The work of D. S. was also supported (in part) by the Yonsei University Research Fund (Post Doctorate Researcher Supporting Program) of 2018 (Project No. 2018-12-0145).
[1] R. Aaij et al. (LHCb Collaboration), Test of lepton universality with $B^{0} \rightarrow K^{* 0} \ell^{+} \ell^{-}$decays, J. High Energy Phys. 08 (2017) 055; Angular analysis of the $B^{0} \rightarrow K^{* 0} \mu^{+} \mu^{-}$decay using $3 \mathrm{fb}^{-1}$ of integrated luminosity, J. High Energy Phys. 02 (2016) 104; Test of Lepton Universality using $B^{+} \rightarrow$ $K^{+} \ell^{+} \ell^{-}$Decays, Phys. Rev. Lett. 113, 151601 (2014).

[2] R. Aaij et al. (LHCb Collaboration), Angular analysis and differential branching fraction of the decay $B_{s}^{0} \rightarrow \phi \mu^{+} \mu^{-}$, J. High Energy Phys. 09 (2015) 179.

[3] A. Abdesselam, and J. Guo (Belle Collaboration), Measurement of the branching ratio of $\bar{B}^{0} \rightarrow D^{*+} \tau^{-} \bar{\nu}_{\tau}$ relative to $\bar{B}^{0} \rightarrow D^{*+} \ell^{-} \bar{\nu}_{\ell}$ decays with a semileptonic tagging method, Measurement 132, 369 (2019); R. Aaij et al. (LHCb Collaboration), Measurement of the Ratio of Branching Fractions $\mathcal{B}\left(\bar{B}^{0} \rightarrow D^{*+} \tau^{-} \bar{\nu}_{\tau}\right) / \mathcal{B}\left(\bar{B}^{0} \rightarrow D^{*+} \mu^{-} \bar{\nu}_{\mu}\right)$, Phys. Rev. Lett. 115, 111803 (2015); Erratum, Phys. Rev. Lett. 115, 159901(E) (2015); M. Huschle et al. (Belle Collaboration), Measurement of the branching ratio of $\bar{B} \rightarrow D^{(*)} \tau^{-} \bar{\nu}_{\tau}$ relative to $\bar{B} \rightarrow D^{(*)} \ell^{-} \bar{\nu}_{\ell}$ decays with hadronic tagging at Belle, Phys. Rev. D 92, 072014 (2015); J. P. Lees et al. (BABAR Collaboration), Measurement of an excess of $\bar{B} \rightarrow D^{(*)} \tau^{-} \bar{\nu}_{\tau}$ decays and implications for charged Higgs bosons, Phys. Rev. D 88, 072012 (2013).

[4] R. Aaij et al. (LHCb Collaboration), Measurement of the Ratio of Branching Fractions $\mathcal{B}\left(B_{c}^{+} \rightarrow J / \psi \tau^{+} \nu_{\tau}\right) /$ $\mathcal{B}\left(B_{c}^{+} \rightarrow J / \psi \mu^{+} \nu_{\mu}\right)$, Phys. Rev. Lett. 120, 121801 (2018).

[5] V. G. Lüth, Semileptonic $B$ meson decays, Annu. Rev. Nucl. Part. Sci. 61, 119 (2011); J. Dingfelder and T. Mannel, Leptonic and semileptonic decays of $B$ mesons, Rev. Mod. Phys. 88, 035008 (2016).

[6] N. Isgur and M. B. Wise, Weak transition form-factors between heavy mesons, Phys. Lett. B 237, 527 (1990); M. Neubert, Heavy quark symmetry, Phys. Rep. 245, 259 (1994); A. V. Manohar and M. B. Wise, Heavy quark physics, Cambridge Monogr. Part. Phys., Nucl. Phys., Cosmol. 10, 1 (2000); N. Uraltsev, in At the Frontier of Particle Physics, Vol. 3, edited by M. Shifman, pp. 1577-1670 (World Scientific, Singapore, 2001), https://doi.org/10.1142/4544; A. G. Grozin, Heavy quark effective theory, Springer Tracts Mod. Phys. 201, 1 (2004).

[7] J. E. Mandula and M. C. Ogilvie, A lattice calculation of the heavy quark universal form factor, Nucl. Phys. B, Proc. Suppl. 34, 480 (1994); T. Bhattacharya and R. Gupta,
Lattice analysis of semileptonic form-factors, Nucl. Phys. B, Proc. Suppl. 47, 481 (1996); J. M. Flynn, in Heavy Flavor Physics, edited by C. Campagnari (World Scientific, Singapore, 1999), pp. 40-51; J. M. Flynn and C. T. Sachrajda, Heavy quark physics from lattice QCD, Adv. Ser. Dir. High Energy Phys. 15, 402 (1998); S. Hashimoto, A. X. El-Khadra, A. S. Kronfeld, P. B. Mackenzie, S. M. Ryan, and J. N. Simone, Lattice QCD calculation of $\bar{B} \rightarrow \mathrm{D} 1 \bar{\nu}$ decay form-factors at zero recoil, Phys. Rev. D 61, 014502 (1999); S. Hashimoto, A. S. Kronfeld, P. B. Mackenzie, S. M. Ryan, and J. N. Simone, Lattice calculation of the zero recoil form-factor of $\bar{B} \rightarrow D^{*} l \bar{\nu}$ : Toward a model independent determination of $\left|V_{\mathrm{cb}}\right|$, Phys. Rev. D 66, 014503 (2002); E. Dalgic, A. Gray, M. Wingate, C. T. H. Davies, G. P. Lepage, and J. Shigemitsu, $B$ meson semileptonic form-factors from unquenched lattice QCD, Phys. Rev. D 73, 074502 (2006); Erratum, Phys. Rev. D 75, 119906(E) (2007); G. M. de Divitiis, E. Molinaro, R. Petronzio, and N. Tantalo, Quenched lattice calculation of the $B \rightarrow D l \nu$ decay rate, Phys. Lett. B 655, 45 (2007); G. M. de Divitiis, R. Petronzio, and N. Tantalo, Quenched lattice calculation of semileptonic heavy-light meson form factors, J. High Energy Phys. 10 (2007) 062; C. M. Bouchard, G. P. Lepage, C. Monahan, H. Na, and J. Shigemitsu, $B_{s} \rightarrow K \ell \nu$ form factors from lattice QCD, Phys. Rev. D 90, 054506 (2014); J. M. Flynn, T. Izubuchi, T. Kawanai, C. Lehner, A. Soni, R. S. Van de Water, and O. Witzel, $B \rightarrow \pi \ell \nu$ and $B_{s} \rightarrow$ $K \ell \nu$ form factors and $\left|V_{u b}\right|$ from $2+1$-flavor lattice QCD with domain-wall light quarks and relativistic heavy quarks, Phys. Rev. D 91, 074510 (2015); H. Na, C. M. Bouchard, G. Peter Lepage, C. Monahan, and J. Shigemitsu (HPQCD Collaboration), $B \rightarrow D l \nu$ form factors at nonzero recoil and extraction of $\left|V_{c b}\right|$, Phys. Rev. D 92, 054510 (2015); Erratum, Phys. Rev. D 93, 119906(E) (2016); J. A. Bailey et al. (MILC Collaboration), $B \rightarrow D \ell v$ form factors at nonzero recoil and $\left|V_{c b}\right|$ from $2+1$-flavor lattice QCD, Phys. Rev. D 92, 034506 (2015); N. H. Christ, X. Feng, A. Juttner, A. Lawson, A. Portelli, and C. T. Sachrajda, First exploratory calculation of the long-distance contributions to the rare kaon decays $K \rightarrow \pi \ell^{+} \ell^{-}$, Phys. Rev. D 94, 114516 (2016); Z. Bai, N. H. Christ, X. Feng, A. Lawson, A. Portelli, and C. T. Sachrajda, Exploratory Lattice QCD Study of the Rare Kaon Decay $K^{+} \rightarrow \pi^{+} \nu \bar{\nu}$, Phys. Rev. Lett. 118, 252001 (2017); Z. Gelzer et al., Semileptonic 
$B$-meson decays to light pseudoscalar mesons on the HISQ ensembles, EPJ Web Conf. 175, 13024 (2018); A. Bazavov et al. (Fermilab Lattice and MILC Collaborations), $B_{s} \rightarrow$ $K \ell \nu$ decay from lattice QCD, arXiv:1901.02561.

[8] P. Ball and R. Zwicky, New results on $B \rightarrow \pi, K, \eta$ decay formfactors from light-cone sum rules, Phys. Rev. D 71, 014015 (2005); A. Khodjamirian, T. Mannel, and N. Offen, Form-factors from light-cone sum rules with B-meson distribution amplitudes, Phys. Rev. D 75, 054013 (2007); S. Faller, A. Khodjamirian, C. Klein, and T. Mannel, $B \rightarrow$ $D(*)$ form factors from QCD light-cone sum rules, Eur. Phys. J. C 60, 603 (2009); A. Khodjamirian, C. Klein, T. Mannel, and N. Offen, Semileptonic charm decays $D \rightarrow$ $\pi l \nu(l)$ and $D \rightarrow K l \nu(l)$ from QCD light-cone sum rules, Phys. Rev. D 80, 114005 (2009); A. Khodjamirian, T. Mannel, N. Offen, and Y.-M. Wang, $B \rightarrow \pi \ell \nu_{l}$ width and $\left|V_{u b}\right|$ from QCD light-cone sum rules, Phys. Rev. D 83, 094031 (2011); A. Bharucha, Two-loop corrections to the $B \rightarrow \pi$ form factor from QCD sum rules on the light-cone and $\left|V_{u b}\right|$, J. High Energy Phys. 05 (2012) 092; Y. M. Wang and Y.L. Shen, QCD corrections to $B$ form factors from light-cone sum rules, Nucl. Phys. B898, 563 (2015); N. Gubernari, A. Kokulu, and D. van Dyk, $B \rightarrow P$ and $B \rightarrow V$ form factors from $B$-meson light-cone sum rules beyond leading twist, J. High Energy Phys. 01 (2019) 150.

[9] M. A. Ivanov, J. G. Korner, S. G. Kovalenko, P. Santorelli, and G. G. Saidullaeva, Form factors for semileptonic, nonleptonic and rare $B\left(B_{s}\right)$ meson decays, Phys. Rev. D 85, 034004 (2012); M. A. Ivanov, J. G. Körner, and C. T. Tran, Exclusive decays $B \rightarrow \ell^{-} \bar{\nu}$ and $B \rightarrow D^{(*)} \ell^{-} \bar{\nu}$ in the covariant quark model, Phys. Rev. D 92, 114022 (2015); M. A. Ivanov and C. T. Tran, Exclusive decays, Phys. Rev. D 92, 074030 (2015); M. A. Ivanov, J. G. Körner, and C. T. Tran, Analyzing new physics in the decays $\bar{B}^{0} \rightarrow D^{(*)} \tau^{-} \bar{\nu}_{\tau}$ with form factors obtained from the covariant quark model, Phys. Rev. D 94, 094028 (2016).

[10] C.S. Kim and D. Sahoo, Probing new physics in $B \rightarrow$ $D \ell^{+} \ell^{-}$decays by using angular asymmetries, Eur. Phys. J. C 77, 582 (2017).

[11] G. Buchalla, A. J. Buras, and M.E. Lautenbacher, Weak decays beyond leading logarithms, Rev. Mod. Phys. 68, 1125 (1996); C. Bobeth, M. Misiak, and J. Urban, Photonic penguins at two loops and $m_{t}$ dependence of $B R\left[B \rightarrow X_{s} l^{+} l^{-}\right]$, Nucl. Phys. B574, 291 (2000); W. Altmannshofer, P. Ball, A. Bharucha, A. J. Buras, D. M. Straub, and M. Wick, Symmetries and asymmetries of $B \rightarrow$ $K^{*} \mu^{+} \mu^{-}$decays in the standard model and beyond, J. High Energy Phys. 01 (2009) 019.

[12] A. Ali, P. Ball, L. T. Handoko, and G. Hiller, A comparative study of the decays $B \rightarrow\left(K, K^{*}\right) \ell^{-} \ell^{+}$in standard model and supersymmetric theories, Phys. Rev. D 61, 074024 (2000); C. Bobeth, T. Ewerth, F. Kruger, and J. Urban, Analysis of neutral Higgs boson contributions to the decays $\bar{B}_{s} \rightarrow \ell^{+} \ell^{-}$and $\bar{B} \rightarrow K \ell^{+} \ell^{-}$, Phys. Rev. D 64, 074014 (2001); C. Bobeth, G. Hiller, and G. Piranishvili, Angular distributions of $\bar{B} \rightarrow \bar{K} \ell^{+} \ell^{-}$decays, J. High Energy Phys. 12 (2007) 040.

[13] J. Gratrex, M. Hopfer, and R. Zwicky, Generalised helicity formalism, higher moments and the $B \rightarrow K_{J_{K}}(\rightarrow K \pi) \bar{\ell}_{1} \ell_{2}$ angular distributions, Phys. Rev. D 93, 054008 (2016).
[14] F. Beaujean, M. Chrząszcz, N. Serra, and D. van Dyk, Extracting angular observables without a likelihood and applications to rare decays, Phys. Rev. D 91, 114012 (2015).

[15] B. Aubert et al. (BABAR Collaboration), Measurements of branching fractions, rate asymmetries, and angular distributions in the rare decays $B \rightarrow K \ell^{+} \ell^{-}$and $B \rightarrow K^{*} \ell^{+} \ell^{-}$, Phys. Rev. D 73, 092001 (2006); R. Aaij et al. (LHCb Collaboration), Angular analysis of charged and neutral $B \rightarrow K \mu^{+} \mu^{-}$decays, J. High Energy Phys. 05 (2014) 082; D. Wang (CMS Collaboration), Angular analyses of $b \rightarrow$ $s \mu^{+} \mu^{-}$transitions at CMS, Proc. Sci., BEAUTY2018 (2018) 045; A. M. Sirunyan et al. (CMS Collaboration), Angular analysis of the decay $\mathrm{B}^{+} \rightarrow \mathrm{K}^{+} \mu^{+} \mu^{-}$in proton-proton collisions at $\sqrt{s}=8 \mathrm{TeV}$, Phys. Rev. D 98, 112011 (2018).

[16] S. L. Glashow, D. Guadagnoli, and K. Lane, Lepton Flavor Violation in B Decays?, Phys. Rev. Lett. 114, 091801 (2015); S. Sahoo and R. Mohanta, Scalar leptoquarks and the rare $B$ meson decays, Phys. Rev. D 91, 094019 (2015); C. J. Lee and J. Tandean, Minimal lepton flavor violation implications of the $b \rightarrow s$ anomalies, J. High Energy Phys. 08 (2015) 123; L. Calibbi, A. Crivellin, and T. Ota, Effective Field Theory Approach to $b \rightarrow s \ell \ell^{(\prime)}, B \rightarrow K^{(*)} v \bar{v}$ and $B \rightarrow D^{(*)} \tau v$ with Third Generation Couplings, Phys. Rev. Lett. 115, 181801 (2015); S. Sahoo and R. Mohanta, Lepton flavor violating $B$ meson decays via a scalar leptoquark, Phys. Rev. D 93, 114001 (2016); C. S. Kim, X. B. Yuan, and Y. J. Zheng, Constraints on a $Z$ boson within minimal flavor violation, Phys. Rev. D 93, 095009 (2016); M. Duraisamy, S. Sahoo, and R. Mohanta, Rare semileptonic $B \rightarrow K(\pi) l_{i}^{-} l_{j}^{+}$ decay in a vector leptoquark model, Phys. Rev. D 95, 035022 (2017); S. Sahoo and R. Mohanta, New physics effects in charm meson decays involving $c \rightarrow u l^{+} l^{-}\left(l_{i}^{\mp} l_{j}^{ \pm}\right)$ transitions, Eur. Phys. J. C 77, 344 (2017); Fayyazuddin, M. J. Aslam, and C. D. Lu, Lepton flavor violating decays of $B$ and $K$ mesons in models with extended gauge group, Int. J. Mod. Phys. A 33, 1850087 (2018); J. H. Sheng, J. J. Song, R. M. Wang, and Y.D. Yang, The lepton flavor violating exclusive $\bar{b} \rightarrow \bar{s} \ell_{i}^{-} \ell_{j}^{+}$decays in SUSY without $R$-parity, Nucl. Phys. B930, 69 (2018); J. H. Sheng, R. M. Wang, and Y.D. Yang, Scalar leptoquark effects in the lepton flavor violating exclusive $b \rightarrow s \ell_{i}^{-} \ell_{j}^{+}$decays, Int. J. Theor. Phys. 58, 480 (2019).

[17] G. Cottin, J. C. Helo, and M. Hirsch, Searches for light sterile neutrinos with multitrack displaced vertices, Phys. Rev. D 97, 055025 (2018); G. Cottin, Reconstructing particle masses in events with displaced vertices, J. High Energy Phys. 03 (2018) 137; S. Antusch, E. Cazzato, and O. Fischer, Sterile neutrino searches via displaced vertices at LHCb, Phys. Lett. B 774, 114 (2017); D. Dercks, J. De Vries, H. K. Dreiner, and Z. S. Wang, $R$-parity violation and light neutralinos at CODEX-b, FASER, and MATHUSLA, Phys. Rev. D 99, 055039 (2019); C. Dib and C.S. Kim, Remarks on the lifetime of sterile neutrinos and the effect on detection of rare meson decays $M^{+} \rightarrow M^{\prime}-\ell^{+} \ell^{+}$, Phys. Rev. D 89, 077301 (2014).

[18] G. Aad et al. (ATLAS Collaboration), Search for long-lived neutral particles decaying into lepton jets in proton-proton collisions at $\sqrt{s}=8 \mathrm{TeV}$ with the ATLAS detector, J. High 
Energy Phys. 11 (2014) 088; V. Khachatryan et al. (CMS Collaboration), Search for long-lived neutral particles decaying to quark-antiquark pairs in proton-proton collisions at $\sqrt{s}=8 \mathrm{TeV}$, Phys. Rev. D 91, 012007 (2015); ATLAS Collaboration, Search for long-lived, massive particles in events with displaced vertices and missing transverse momentum in $13 \mathrm{TeV} p p$ collisions with the ATLAS detector, CERN Report No. ATLAS-CONF-2017026, 2017; R. Aaij et al. (LHCb Collaboration), Search for massive long-lived particles decaying semileptonically in the LHCb detector, Eur. Phys. J. C 77, 224 (2017); J. P. Chou, D. Curtin, and H. J. Lubatti, New detectors to explore the lifetime frontier, Phys. Lett. B 767, 29 (2017); C. Alpigiani (MATHUSLA Collaboration), Ultra long-lived particles with MATHUSLA, Proc. Sci., ALPS2018 (2018) 033; V. V. Gligorov, S. Knapen, M. Papucci, and D. J. Robinson, Searching for long-lived particles: A compact detector for exotics at LHCb, Phys. Rev. D 97, 015023 (2018); J. L. Feng, I. Galon, F. Kling, and S. Trojanowski, ForWard Search ExpeRiment at the LHC, Phys. Rev. D 97, 035001 (2018).

[19] C. S. Kim, S. C. Park, K. Wang, and G. Zhu, Invisible Higgs decay with $B \rightarrow K \nu \bar{\nu}$ constraint, Phys. Rev. D 81, 054004 (2010). 